\title{
Primary Semiclassical Kinetic Hydrogen Isotope Effects in Identity \\ Carbon-to-Carbon Proton and Hydride Transfer Reactions, An ab initio and DFT Computational Study
}

\author{
Scott Gronert* and James R. Keeffe* \\ Contribution from the Department of Chemistry and Biochemistry, San Francisco State \\ University, 1600 Holloway Avenue, San Francisco, California 94132 \\ E-mailsgronert@sfsu.edu; keeffe@sfsu.edu
}

Supporting Information Index

1. Reference $24 \quad$ S1

2. Table S1 S2 - S6

3. Table S2 S7 - S58

24. Frisch, M. J.; Trucks, G. W.; Schlegel, H. B.; Scuseria, G. E.; Robb, M. A.; Cheeseman, J. R.; Montgomery, J. A.; Vreven, T.; Kudin, K. N.; Burant, J. C.; Millam, J. M.; Iyengar, S. S.; Tomasi, J.; Barone, V.; Mennucci, B.; Cossi, M.; Scalmani, G.; Rega, N.; Petersson, G. A.; Nakatsuji, H.; Hada, M.; Ehara, M.; Toyota, K.; Fukuda, R.; Hasegawa, J.; Ishida, M.; Nakajima, T.; Honda, Y.; Kitao, O.; Nakai, H.; Klene, M.; Li, X.; Knox, J. E.; Hratchian, H. P.; Cross, J. B.; Adamo, C.; Jaramillo, J.; Gomperts, R.; Stratmann, R. E.; Yazyev, O.; Austin, A. J.; Cammi, R.; Pomelli, C.; Ochterski, J. W.; Ayala, P. Y.; Morokuma, K.; Voth, G. A.; Salvador, P.; Dannenberg, J. J.; Zakrzewski, V. G.; Dapprich, S.; Daniels, A. D.; Strain, M. C.; Farkas, O.; Malick, D. K.; Rabuck, A. D.; Raghavachari, K.; Foresman, J. B.; Ortiz, J. V.; Cui, Q.; Baboul, A. G.; Clifford, S.; Cioslowski, J.; Stefanov, B. B.; Liu, G.; Liashenko, A.; Piskorz, P.; Komaromi, I.; Martin, R. L.; Fox, D. J.; Keith, T.; Al-Laham, M. A.; Peng, C. Y.; Nanayakkara, A.; Challacombe, M.; Gill, P. M. W.; Johnson, B.; Chen, W.; Wong, M. W.;

Gonzalez, C.; Pople, J. A. Gaussian 03, Revision B04; Gaussian, Inc.: Pittsburgh, PA, 2003. 
Table S1. Electronic Energies, ${ }^{a}$ Zero-point Vibrational Energies, ${ }^{\mathrm{b}}$ and Transition State Imaginary Frequencies ${ }^{\mathrm{c}}$ for the Intermolecular $^{\mathrm{d}}$ and Intramolecular ${ }^{\mathfrak{e}}$ Hydride and Proton Transfer Reactions in this Study.

\begin{tabular}{|c|c|c|c|c|c|c|c|c|c|}
\hline \multirow[b]{2}{*}{ System $^{\mathrm{f}}$} & \multicolumn{3}{|c|}{$\mathrm{HF} / 6-311+\mathrm{G}^{* *}$} & \multicolumn{3}{|c|}{$\mathrm{MP} 2 / 6-311+\mathrm{G}^{* *}$} & \multicolumn{3}{|c|}{ B3LYP/6-311++G** } \\
\hline & Energy & ZPVE & iv & Energy & ZPVE & iv & Energy & ZPVE & iv \\
\hline & & & & Identity Proton & Transfer & & & & \\
\hline 1d methane & -40.209086 & 29.45 & & -40.379526 & 28.51 & & -40.5339573 & 27.96 & \\
\hline 1 a methyl ${ }^{-}$ & -39.5180657 & 18.69 & & -39.697972 & 18.28 & & -39.8562124 & 17.85 & \\
\hline $1 \mathrm{ts}$ & -79.6943302 & 45.51 & 1867,1342 & -80.0626481 & 44.18 & 1502,1080 & -80.378763 & 43.51 & 1446,1040 \\
\hline 2d ethane & -79.2518615 & 49.33 & & -79.5714788 & 47.56 & & -79.8565732 & 46.63 & \\
\hline $\mathbf{2 a}$ ethyl $^{-}$ & -78.5539102 & 38.16 & & -78.8858536 & 36.90 & & -79.1443409 & 35.87 & \\
\hline 2 ts inter & -157.7743323 & 84.34 & 1839,1325 & -158.4461537 & 81.35 & 1393,1008 & -159.0187154 & 79.53 & 1427,1030 \\
\hline 12 ts intra & -78.459124 & 34.86 & 1950,1449 & -78.8071521 & 33.66 & 1739,1295 & -79.0966864 & 32.78 & 1712,1275 \\
\hline 3d propane & -118.2961382 & 68.39 & & -118.7670793 & 65.79 & & -119.1811288 & 64.56 & \\
\hline 3a propyl ${ }^{-}$ & -117.6022129 & 57.23 & & -118.0891913 & 55.11 & & -118.5080536 & 53.92 & \\
\hline 3 ts inter & -235.8665408 & 122.32 & 1857,1334 & -236.8443353 & 117.64 & 1425,1028 & -237.6762582 & 115.59 & 1447,1044 \\
\hline 13ts intra & -117.5272528 & 53.56 & 2114,1536 & -118.0332157 & 51.40 & 1743,1267 & -118.4492499 & 50.13 & 1780,1292 \\
\hline 14 butyl $^{-}$ & -156.6467674 & 76.15 & & -157.2850211 & 73.16 & & -157.8304357 & 71.68 & \\
\hline 14 ts intra & -156.5999145 & 72.75 & 1906,1380 & -157.2572674 & 69.73 & 1498,1085 & -157.800957 & 68.34 & 1533,1111 \\
\hline 15 pentyl $^{-}$ & -195.6910626 & 95.06 & & -196.4809551 & 91.21 & & -197.1558783 & 89.47 & \\
\hline 15 ts intra & -195.6472104 & 91.48 & 1941,1403 & -196.45507 & 87.49 & 1551,1122 & -197.1279756 & 85.76 & 1562,1129 \\
\hline $\mathbf{4 d ~} \mathrm{HCF}_{2} \mathrm{CF}_{2}^{+}$ & -473.8294377 & 23.38 & & -474.900251 & 21.78 & & -475.9201168 & 20.98 & \\
\hline $\mathbf{4 a} \mathrm{CF}_{2}=\mathrm{CF}_{2}$ & -473.5577336 & 15.09 & & -474.6379119 & 13.60 & & -475.6659197 & 12.38 & \\
\hline 4 ts & -947.3274708 & 35.26 & 1788,1430 & -949.5208829 & NA & NA & -951.5764143 & 3202 & 958,798 \\
\hline 5d methanol & -115.0804344 & 34.37 & & -115.4449742 & 32.75 & & -115.7649993 & 32.02 & \\
\hline $5 \mathbf{a}$ methoxide ${ }^{-}$ & -114.4436008 & 23.81 & & -114.8197037 & 22.63 & & -115.1468137 & 221.65 & \\
\hline 5cx complex & -229.5600901 & 59.55 & & -230.3101212 & 55.55 & & -230.9540468 & 53.99 & \\
\hline 5 ts & -229.556032 & 56.62 & 1214,877 & -230.3101042 & 54.25 & 206,163 & -230.9539393 & 52.70 & 356,267 \\
\hline
\end{tabular}


Table S1 (cont.). Electronic Energies, ${ }^{a}$ Zero-point Vibrational Energies, ${ }^{b}$ and Transition State Imaginary Frequencies ${ }^{\mathrm{c}}$ for the Intermolecular $^{\mathrm{d}}$ and Intramolecular ${ }^{\mathrm{e}}$ Hydride and Proton Transfer Reactions in this Study.

\begin{tabular}{|c|c|c|c|c|c|c|c|c|c|}
\hline \multirow[b]{2}{*}{ System $^{\mathrm{f}}$} & \multicolumn{3}{|c|}{$\mathrm{HF} / 6-311+\mathrm{G}^{* *}$} & \multicolumn{3}{|c|}{$\mathrm{MP} 2 / 6-311+\mathrm{G}^{* *}$} & \multicolumn{3}{|c|}{ B3LYP/6-311++G** } \\
\hline & Energy & ZPVE & iv & Energy & ZPVE & iv & Energy & ZPVE & iv \\
\hline 6d $\mathrm{HCF}_{3}$ & -336.8743272 & 17.38 & & -337.6314967 & 16.16 & & -338.3492073 & 15.66 & \\
\hline $\mathbf{6 a} \mathrm{CF}_{3}^{-}$ & -336.245717 & 6.58 & & -337.0123327 & 5.89 & & -337.7401812 & 5.43 & \\
\hline 6ts & -673.1171606 & 21.13 & 1671,1198 & -674.6536591 & 19.28 & 1333,965 & -676.1025408 & 18.49 & 1198,870 \\
\hline 7d $\mathrm{HCCl}_{3}$ & -1416.9606222 & 13.67 & & -1417.5272952 & 12.97 & & -1419.3797089 & 12.40 & \\
\hline $7 \mathbf{a ~} \mathrm{CCl}_{3}^{-}$ & -1416.3693618 & 3.63 & & -1416.9313181 & 3.64 & & -1418.8025188 & 3.05 & \\
\hline 7 ts & -2833.3305566 & 14.70 & 1610,1143 & -2834.4878701 & 14.10 & 927,671 & -2838.1958011 & 12.89 & 1071,768 \\
\hline $\mathbf{8 d ~} \mathrm{CH}_{3} \mathrm{CHO}$ & -152.9616541 & 37.10 & & -153.4494336 & 35.17 & & -153.8822521 & 34.63 & \\
\hline $8 \mathbf{a ~} \mathrm{CH}_{2} \mathrm{CHO}^{-}$ & -152.3509179 & 28.04 & & -152.8504986 & 26.14 & & -153.2899434 & 26.05 & \\
\hline 8 ts & -305.2864972 & 62.95 & 1861,1390 & -306.2996032 & 59.99 & 1179,892 & -307.1737154 & 59.10 & 1166,883 \\
\hline 9d $\mathrm{CH}_{3} \mathrm{CN}$ & -131.9627077 & 30.32 & & -132.4109416 & 28.46 & & -132.7962006 & 28.34 & \\
\hline 9a $\mathrm{CH}_{2} \mathrm{CN}^{-}$ & -131.3430255 & 20.13 & & -131.798835 & 19.20 & & -132.1918599 & 18.83 & \\
\hline 9ts & -263.2985694 & 48.54 & 1766,1280 & -264.2218903 & 45.58 & 1222,887 & -265.0001835 & 45.57 & 1186,866 \\
\hline $10 d \mathrm{CH}_{3} \mathrm{NO}_{2}$ & -243.7360181 & 33.83 & & -244.4782926 & 31.85 & & -245.0916543 & 31.15 & \\
\hline $\mathbf{1 0 a} \mathrm{CH}_{2} \mathrm{NO}_{2}^{-}$ & -243.1474917 & 24.48 & & -243.8908651 & 22.61 & & -244.516498 & 22.30 & \\
\hline 10 ts & -486.8691914 & 56.09 & 1805,1342 & -488.3794297 & 53.00 & 1188,903 & -489.6196726 & 51.98 & 1135,861 \\
\hline 11d $\mathrm{CH}_{3} \mathrm{C} \equiv \mathrm{CH}$ & -115.8976258 & 37.33 & & -116.3165964 & 34.94 & & -116.6928501 & 34.82 & \\
\hline 11a $\mathrm{CH}_{3} \mathrm{C} \equiv \mathrm{C}^{-}$ & -115.2716658 & 28.85 & & -115.6950624 & 27.09 & & -116.0721138 & 26.84 & \\
\hline 11 ts & -231.1607571 & 64.13 & 1718, NA & -232.0152451 & 58.81 & 1328,948 & -232.7696297 & 59.50 & 1292,921 \\
\hline & & & & on-identity Proto & on Tran & fers & & & \\
\hline 16d $\mathrm{CH}_{3} \mathrm{CHO}$ & -152.9616541 & 37.10 & & -153.4494336 & 35.17 & & -153.8822521 & 34.63 & \\
\hline $16 \mathbf{a} \mathrm{CH}_{2} \mathrm{CN}^{-}$ & -131.3430255 & 20.13 & & -131.798835 & 19.20 & & -132.1918599 & 18.83 & \\
\hline $16 \mathrm{ts}$ & -284.2920671 & 55.67 & 1787,1313 & -285.2590376 & 52.75 & 977,743 & -286.0862702 & 52.28 & 996,750 \\
\hline
\end{tabular}


Table S1 (cont.). Electronic Energies, ${ }^{a}$ Zero-point Vibrational Energies, ${ }^{b}$ and Transition State Imaginary Frequencies ${ }^{\mathrm{c}}$ for the Intermolecular ${ }^{\mathrm{d}}$ and Intramolecular ${ }^{\mathrm{e}}$ Hydride and Proton Transfer Reactions in this Study.

\begin{tabular}{|c|c|c|c|c|c|c|c|c|c|}
\hline \multirow[b]{2}{*}{ System $^{\mathrm{f}}$} & \multicolumn{3}{|c|}{$\mathrm{HF} / 6-311+\mathrm{G}^{* *}$} & \multicolumn{3}{|c|}{$\mathrm{MP} 2 / 6-311+\mathrm{G}^{* *}$} & \multicolumn{3}{|c|}{ B3LYP/6-311++G** } \\
\hline & Energy & ZPVE & iv & Energy & ZPVE & iv & Energy & ZPVE & iv \\
\hline 17d $\mathrm{CH}_{3} \mathrm{CN}$ & -131.9627077 & 30.32 & & -132.4109416 & 28.46 & & -132.7962006 & 28.34 & \\
\hline $17 \mathbf{a ~} \mathrm{CH}_{2} \mathrm{CHO}^{-}$ & -152.3509179 & 28.04 & & -152.8504986 & 26.14 & & -153.2899434 & 26.05 & \\
\hline 17 ts & -284.2920671 & 55.67 & 1787,1313 & -285.2590376 & 52.75 & 977,743 & -286.0862702 & 52.28 & 996,750 \\
\hline $\mathbf{1 8 d ~} \mathrm{CH}_{3} \mathrm{NO}_{2}$ & -243.7360181 & 33.83 & & -244.4782926 & 31.85 & & -245.0916543 & 31.15 & \\
\hline $\mathbf{1 8 a ~} \mathrm{CH}_{2} \mathrm{CN}^{-}$ & -131.3430255 & 20.13 & & -131.798835 & 19.20 & & -132.1918599 & 18.83 & \\
\hline $18 t s$ & -375.0839748 & 52.33 & 1720,1265 & -376.3004353 & 49.45 & 797,623 & -377.3105081 & 48.85 & 869,663 \\
\hline 19d $\mathrm{CH}_{3} \mathrm{CN}$ & -131.9627077 & 30.32 & & -132.4109416 & 28.46 & & -132.7962006 & 28.34 & \\
\hline 19a $\mathrm{CH}_{2} \mathrm{NO}_{2}^{-}$ & -243.1474917 & 24.48 & & -243.8908651 & 22.61 & & -244.516498 & 22.30 & \\
\hline 19 ts & -375.0839748 & 52.33 & 1720,1265 & -376.3004353 & 49.45 & 797,623 & -377.3105081 & 48.85 & 869,663 \\
\hline \multicolumn{10}{|c|}{$\underline{\text { Identity Hydride Transfers to Carbonyls }}$} \\
\hline $\operatorname{20d} \mathrm{HCO}_{2}^{-}$ & -188.2571919 & 13.78 & & -188.8024544 & 12.69 & & -189.2728006 & 12.48 & \\
\hline $\mathbf{2 0 a} \mathrm{CO}_{2}$ & -187.6915605 & 8.05 & & -188.206558 & 7.26 & & -188.6469148 & 7.33 & \\
\hline 20 ts & -375.9081883 & 19.60 & 1297 & -376.9974175 & 18.60 & 285,272 & -377.9148381 & 18.30 & 393,366 \\
\hline 21d $\mathrm{HCOS}^{-}$ & -510.9126329 & 12.51 & & -511.3829784 & 11.63 & & -512.2436938 & 11.41 & \\
\hline 21a COS & -510.3171264 & 6.19 & & -510.7721828 & 6.19 & & -511.600125 & 5.74 & \\
\hline 21ts (anti) & -1021.1798577 & 16.25 & 1361,1151 & -1022.1430766 & 16.37 & $\mathrm{NA}^{\mathrm{g}}$ & -1023.831373 & 15.42 & 466,431 \\
\hline 22ts (syn) & -1021.181071 & 16.46 & 1296,1094 & -1022.1433691 & 16.51 & $\mathrm{NA}^{\mathrm{g}}$ & -1023.8317272 & 15.51 & 446,414 \\
\hline 23d $\mathrm{CH}_{3} \mathrm{O}^{-}$ & -114.4436008 & 23.81 & & -114.8197037 & 22.63 & & -115.1468137 & 21.65 & \\
\hline 23a $\mathrm{CH}_{2} \mathrm{O}$ & -113.9028018 & 18.03 & & -114.2416093 & 16.90 & & -114.5418486 & 16.64 & \\
\hline 23 ts & -228.3298491 & 42.07 & 1305,1091 & -229.0732106 & 40.44 & 62,60 & -229.7080002 & 38.74 & 168,149 \\
\hline 24d $\mathrm{H}_{2} \mathrm{CONH}_{2}^{-}$ & -169.510595 & 37.05 & & -170.0704528 & 34.90 & & -170.5402561 & 34.02 & \\
\hline 24a $\mathrm{HCONH}_{2}$ & -168.988223 & 30.51 & & -169.5054444 & 28.94 & & -169.9548504 & 28.40 & \\
\hline 24ts $(\mathrm{R}, \mathrm{R})$ & -338.467979 & 67.64 & $1500, \mathrm{NA}$ & -339.5760671 & 63.75 & 507,467 & 340.4931667 & 62.50 & 726,639 \\
\hline
\end{tabular}


25ts $(\mathrm{R}, \mathrm{S})$

$-338.4645266 \quad 67.35 \quad 1489$, NA

26d methane $\quad-40.2090866 \quad 29.45$

26a methyl ${ }^{+} \quad-39.2436957 \quad 20.76$

26cx complex ${ }^{\mathrm{g}} \quad-79.4865644 \quad 54.73$ $\begin{array}{lll}-339.5707102 & 63.53 & 208,198\end{array}$

Other Identity Hydride Transfers

$\begin{array}{ll}-40.379526 & 28.51 \\ -39.3563165 & 20.07 \\ -79.8013677 & 53.16\end{array}$

$-340.4893718$

62.12

499,438 $\begin{array}{ll}-40.5339573 & 27.96\end{array}$

$-39.4914723 \quad 19.55$

$-80.0910899 \quad 51.72$ 
Table S1 (cont.). Electronic Energies, ${ }^{a}$ Zero-point Vibrational Energies, ${ }^{b}$ and Transition State Imaginary Frequencies ${ }^{\mathrm{c}}$ for the Intermolecular ${ }^{\mathrm{d}}$ and Intramolecular ${ }^{\mathrm{e}}$ Hydride and Proton Transfer Reactions in this Study.

\begin{tabular}{|c|c|c|c|c|c|c|c|c|c|}
\hline \multirow[b]{2}{*}{ System $^{\mathrm{f}}$} & \multicolumn{3}{|c|}{$\mathrm{HF} / 6-311+\mathrm{G}^{* *}$} & \multicolumn{3}{|c|}{$\mathrm{MP} 2 / 6-311+\mathrm{G}^{* *}$} & \multicolumn{3}{|c|}{ B3LYP/6-311++G** } \\
\hline & Energy & ZPVE & iv & Energy & ZPVE & iv & Energy & ZPVE & iv \\
\hline $\begin{array}{l}\mathbf{2 7 d} 1,4 \text {-dihydro } \\
\text { pyridine }\end{array}$ & NA & NA & & NA & NA & & -249.5311248 & 69.36 & \\
\hline 27a pyridinium & NA & NA & & NA & NA & & -248.7191386 & 64.36 & \\
\hline $27 \mathrm{ts}$ & NA & NA & NA & NA & NA & & -498.2453597 & 132.00 & 897,754 \\
\hline 28d $\mathrm{HCF}_{3}$ & -336.8743272 & 17.38 & & -337.6314967 & 16.16 & & -338.3492073 & 15.66 & \\
\hline $\mathbf{2 8 a} \mathrm{CF}_{3}^{+}$ & -335.9017426 & 10.03 & & -336.6339751 & 9.28 & & -337.3276659 & 9.04 & \\
\hline 28ts & -672.7181097 & 24.96 & 1024,938 & -674.2353795 & 23.56 & 242,234 & -675.6549548 & 22.53 & 231,220 \\
\hline 29d $\mathrm{HCCl}_{3}$ & -1416.9606222 & 213.67 & & -1417.5272952 & 12.97 & & -1419.3797089 & 12.40 & \\
\hline 29a $\mathrm{CCl}_{3}{ }^{+}$ & -1416.0345295 & 5.81 & & -1416.5899972 & 5.65 & & -1418.4213257 & 5.37 & \\
\hline $29 t s$ & -2832.9359056 & 516.09 & 1272,1061 & $-2834.1092492^{1}$ & $2^{h} 16.73$ & & -2837.7786079 & 14.97 & 718,627 \\
\hline 30d $\mathrm{HCF}_{2} \mathrm{CF}_{2}^{-}$ & -474.1643934 & 20.85 & & -475.2737374 & 19.42 & & -476.3223695 & 18.61 & \\
\hline $\mathbf{3 0 a} \mathrm{CF}_{2}=\mathrm{CF}_{2}$ & -473.5577336 & 15.09 & & -474.6379119 & 13.60 & & -475.6659197 & 12.38 & \\
\hline 30ts & -947.6320644 & 32.58 & 1713,1459 & -949.8780841 & NA & NA & -951.9582775 & 29.01 & 976,804 \\
\hline $31 \mathrm{HCF}_{2} \mathrm{CF}_{2}^{+}$ & -473.8294377 & 23.38 & & -474.900251 & 21.78 & & -475.9201168 & 20.98 & \\
\hline 31ts intra & -473.7765814 & 21.22 & 1048,913 & -474.8571135 & 19.58 & 949,825 & -475.8824522 & 18.93 & 952,818 \\
\hline $32 \mathrm{Me}_{2} \mathrm{CHCMe}_{2}$ & $2^{+}-234.577245$ & 115.90 & & -235.4602732 & 110.55 & & -236.2515879 & 109.00 & \\
\hline 32ts intra & -234.5628044 & 114.65 & 478,405 & -235.453 .1227 & 109.70 & $\mathrm{NA}^{\mathrm{h}}$ & -236.2410684 & 107.54 & 437,367 \\
\hline
\end{tabular}

${ }^{a}$ Hartrees. The compound numbering system is keyed to the reactions listed in Tables 1, 2, and 3 in the manuscript. The indictors $\mathbf{d}, \mathbf{a}, \mathbf{t s}$, and $\mathbf{c x}$ refer to the hydrogen donor, acceptor, transition state, and complex, respectively. ${ }^{\mathrm{b}}$ For the protium isotopomers, $\mathrm{kcal} / \mathrm{mol}{ }^{\mathrm{c}} \mathrm{cm}^{-1}$. All transition states have a single imaginary frequency corresponding to the hydrogen transfer coordinate. The imaginary frequency for $\mathrm{H}$ transfer is given first, then that for D transfer. ${ }^{\mathrm{d}}$ All transfers are intermolecular (inter) except as otherwise identified. ${ }^{\mathrm{e}}$ Intramolecular transfers are identified as (intra). ${ }^{\mathrm{f}}$ The hydrogen donor is listed first followed by the acceptor, then the transition state (ts) or complex (cx). ${ }^{\mathrm{g}}$ These systems produced addition complexes rather than transition states. ${ }^{\mathrm{h}}$ The hydride transfer between trichloromethyl cation and chloroform produces a complex, not a transition state, at the MP2 level. The 1,2-hydride shift in the tetramethylethyl cation produces, at the MP2 level, a symmetrically hydrogen-bridged cation with no imaginary frequency. This cation is an isomer of the optimized cation, with an enthalpy $3.7 \mathrm{kcal} / \mathrm{mol}$ higher. 
Table S2. Cartesian Coordinates for Compounds in this Study.

The numbering system is keyed to Table $\mathrm{S} 1$ and to Tables 1, 2, and 3 in the manuscript. Indicators d, a, ts, cx refer to hydrogen donors, acceptors, transition states, and bimolecular complexes, respectively. The terms inter and intra denote intermolecular and intramolecular transition states, respectively. HF stands for HF/6-311+G**, MP2 stands for MP2/6-311+G**, and DFT stands for B3LYP/6-211++G**.

\section{$\underline{\text { Identity Proton Transfers }}$}

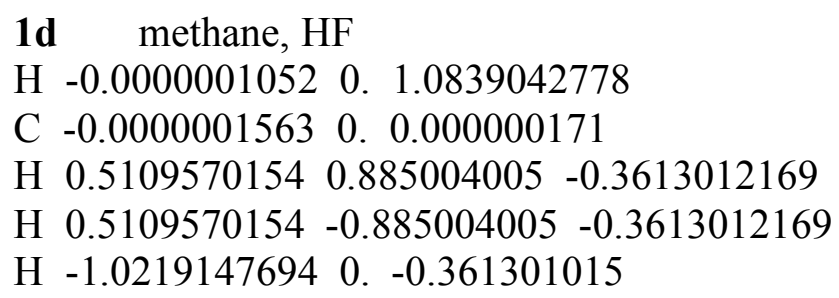

$1 a \quad \mathrm{CH} 3-$, methyl anion, MP2 H $0.5194757675 \quad 0.89975842260 .2491263413$

C 0.0 .0 .1245631707

H $0.5194757675 \quad-0.8997584226 \quad 0.2491263413$

H -1.0389515350 .0 .2491263413$

$1 a \quad \mathrm{CH} 3$ - methyl anion, DFT

H $0.1 .038951535-0.2491263413$

C 0. 0.0 .1245631707 
$\begin{array}{llll}\text { H } & -0.8997584226 & -0.5194757675 & -0.2491263413 \\ \text { H } & 0.8997584226 & -0.5194757675 & -0.2491263413\end{array}$

1 ts $\mathrm{CH} 4$, proton transfer ts, $\mathrm{HF}$

H $-0.8878218165 \quad-0.51265456391 .8548905976$

C 0. 0. 1.4573690987

H -0.00006096751 .02520352911 .8548905976$

H 0. 0. 0 .

H $0.8878827839-0.51254896521 .8548905976$

C 0. 0. -1.4573690987

H $-0.88782181650 .5126545639-1.8548905976$

$\mathrm{H}-0.0000609675-1.0252035291-1.8548905976$

H $0.88788278390 .5125489652-1.8548905976$

1ts $\mathrm{CH} 4$, proton transfer ts, MP2

$\mathrm{H} \quad-0.892324715 \quad-0.5208380788 \quad 1.8247001562$

C $\quad$ 0. 0.1 .4372090579

$\mathrm{H} \quad-0.00489665 \quad 1.033194911 \quad 1.8247001562$

$\mathrm{H} \quad 0.0 .0$.

H $\quad 0.897221365-0.51235683221 .8247001562$

C $\quad 0.0$. -1.4372090579

$\mathrm{H} \quad-0.892324715 \quad 0.5208380788-1.8247001562$

$\mathrm{H} \quad-0.00489665 \quad-1.033194911-1.8247001562$

$\mathrm{H} \quad \begin{array}{llll}0.897221365 & 0.5123568322 & -1.8247001562\end{array}$

1 ts $\mathrm{CH} 4$, proton transfer ts, DFT

H $-0.920705282-0.4735697704 \quad 1.8250394421$

C 0. 0. 1.4452559665

H 0.05022918941 .03413904881 .8250394421

H 0. 0. 0 .

H $0.8704760926-0.56056927841 .8250394421$

C $0.0 .-1.4452559665$

H $-0.9207052820 .4735697704 \quad-1.8250394421$

H $0.0502291894-1.0341390488-1.8250394421$

Н $0.8704760926 \quad 0.5605692784-1.8250394421$

2d ethane, $\mathrm{HF}$

H $0.37622605260 .6516415714 \quad-1.3397305465$

$\begin{array}{llll}\text { C } & 0.3598054079 & 0.6232008818 & -0.2544207526\end{array}$

H $1.3885187175 \quad 0.651641763 \quad 0.0918681444$

C $-0.3598054079 \quad-0.6232008818 \quad 0.2544207526$

H $-0.1299205589 \quad 1.52831339340 .0918678087$ 

H $0.1299205589-1.5283133934-0.0918678087$
H $-1.3885187175-0.651641763-0.0918681444$
H $-0.3762260526-0.65164157141 .3397305465$
2d ethane, MP2
H $0.376223 \quad 0.651637-1.348067$
$\begin{array}{llll}\text { C } & 0.36058 & 0.624543 & -0.254969\end{array}$
H 1.3963780 .6516370 .09465
C $-0.36058-0.624543 \quad 0.254969$
$\begin{array}{llll}\mathrm{H} & -0.133854 & 1.535117 & 0.094649\end{array}$
H $0.133854-1.535117-0.094649$
H $-1.396378-0.651637-0.09465$
H $-0.376223-0.651637 \quad 1.348067$
2d ethane, DFT
H $0.37881131660 .6560888936-1.3482542808$
$\begin{array}{llll}\text { C } & 0.3608774325 & 0.6250743817 & -0.2551963861\end{array}$
H 1.39740445350 .65608348920 .0922761762
C $-0.3608774325 \quad-0.62507438170 .2551963861$
H $-0.1305197676 \quad 1.5382229570 .0922966214$
H $0.1305197676-1.538222957-0.0922966214$
H $-1.3974044535-0.6560834892-0.0922761762$
H $-0.3788113166-0.65608889361 .3482542808$
2a ethyl-, HF
H 0.39107328280 .5699287018 - 1.34423142
$\begin{array}{llll}\text { C } & 0.3546076273 & 0.5402719022 & -0.2507955163\end{array}$
H 1.39766231160 .57005883250 .0792968678
C $-0.3452172581 \quad-0.73577566920 .2441733794$
H $-0.080545828 \quad 1.51412125890 .0568161594$
H $-0.3727068209-0.7404772011 \quad 1.344549208$
H $-1.3918251594-0.74060899-0.0966979911$

2a ethyl-, MP2

H $0.39202141180 .5635917059-1.3503958824$

C $0.35413941180 .5405957059 \quad-0.2504558824$

H 1.40378841180 .56370570590 .0804331176

C $-0.3481087745-0.73164429410 .2462178542$

H -0.07236658821 .52988070590 .0510161176$

H $-0.3641125882-0.7553762941 \quad 1.3515161176$

H $-1.3955155882-0.7555102941-0.1071428824$ 

2a ethyl-, DFT
H $0.39272541920 .5636170852-1.349400231$
$\begin{array}{llll}\text { C } & 0.3547017654 & 0.5356881926 & -0.2508622947\end{array}$
H $1.40308615750 .5637489043 \quad 0.0794664867$
C $-0.3482068514-0.72643388640 .2462862089$
H $-0.0698927321 \quad 1.54261907640 .0492807744$
H $-0.3697707564-0.7626898717 \quad 1.3490794133$
H $-1.3951175718-0.762821031-0.100969929$
2ts inter ethyl- + ethane, ts, HF
H $-0.6147769116-2.4858537888 \quad 1.5937503655$
C $-1.1192508838-2.2172259772 \quad 0.6616388821$
H $-0.4363398986-2.5016196684-0.1434938204$
C $-1.4016109901-0.7115920167 \quad 0.6189807008$
H $-1.9924210783-2.8787600863 \quad 0.5779589307$
H $-0.0933764054-0.0877881740 .7476164208$
H - $-2.0849983216-0.4333359187 \quad 1.4336285525$
H $-1.9048089715-0.4492940977-0.3223410947$
C 1.21503406610 .53602728650 .8762300089
H $1.7181849976 \quad 0.274076852 \quad 1.8176788392$
C $0.9327722293 \quad 2.0416721637 \quad 0.8329763153$
H $1.89846337890 .2574360943 \quad 0.0617253405$
H 1.80596955742 .70320639190 .9164116348
H 0.2498691332 .32641996761 .637995722
H $0.42831980652 .3099810411-0.0992407647$

2ts inter ethyl- + ethane, ts, MP2

H $-0.2775983244-2.38765333410 .5325463698$

C $-0.9665430987-2.0165424608-0.2428218098$

H $-0.4133944463-2.0861773688-1.1918378727$

C $-1.3329752048-0.55946476580 .0439346571$

H $-1.795843125-2.7431129282-0.3061223822$

H -0.01857897380 .00164352730 .1809293723$

H - $1.9029147632-0.47349873680 .9850655011$

H $-1.9485255398 \quad-0.1389286847 \quad-0.7691896896$

C $1.2972646147 \quad 0.5626648713 \quad 0.3035007292$

H $1.6638351927 \quad 0.4949033107 \quad 1.3422414769$

C $0.99954120042 .0135939378-0.0782707462$ 
H $2.0644506057 \quad 0.1284977983 \quad-0.3597087163$

H 1.82568707392 .74047727740 .0153943603

H 0.16785471392 .39739510420 .5337482564

H $0.65130251642 .0649445401-1.1211236577$

2ts inter ethyl- + ethane, ts, DFT

H $-0.4310046233-2.4570604387 \quad 0.7062566725$

C $-1.0144413097-2.1139879662-0.1630142728$

H $-0.3811269122-2.3092153421-1.0425983224$

C $-1.3179850479-0.6194905886-0.0451015056$

H $-1.8784236049-2.8009575217-0.24576959$

H -0.00893392590 .00084849260 .0864159057$

H $-1.9370660426-0.40576696620 .8429760071$

H $-1.8601161563-0.249944845 \quad-0.9321155426$

C 1.30074439870 .62103228410 .2124752101

H $1.7259394742 \quad 0.424812651 \quad 1.2116390041$

C 1.03041892552 .11255487060 .0079119927

H $2.0111565723 \quad 0.2362552822 \quad-0.5388745965$

H 1.89454549692 .79945039280 .0897764943

H 0.28324423742 .47050849220 .7340837049

H $0.58936368472 .2904182032-0.9854182838$

12ts intra ethyl anion, intramolecular $\mathrm{H}+\mathrm{ts}$, $\mathrm{HF}$ H $0.000189989-0.0002361916-1.023185031$ H $1.268012855-0.90317470350 .0899562712$ C $-0.8018811898-0.0695536038-0.034771681$

C $0.80189439950 .0695388669-0.0345022151$

H $-1.2249886729 \quad-0.82272608760 .6298532851$

H $1.22475254190 .8230099106 \quad 0.6299429735$

H - 1.2680459710 .90321549390 .0890758779

12ts intra ethyl anion, intramolecular $\mathrm{H}+\mathrm{ts}$, MP2 H $0.0001960954-0.000236724-1.047604974$ H $1.2838757923-0.89080469830 .0073046999$ C $-0.7925722515-0.0864665566-0.0274999209$ $\begin{array}{llll}\text { C } & 0.7925824912 & 0.0864541868 & -0.0272423604\end{array}$ H $-1.2455636101 \quad-0.80044685880 .6811140637$ H 1.24530866730 .80075470910 .6812183763 H -1.28387838290 .89080779080 .0064215219$

12ts intra ethyl anion, intramolecular $\mathrm{H}+\mathrm{ts}$, DFT H $0.0001970283-0.0002379077-1.0527821569$

H $1.2923370615-0.8851605206-0.0048943766$

C $-0.7882757806-0.0851002096-0.0249020833$

C $0.78828505730 .0850890163-0.024645492$

H $-1.2573953254-0.79675141610 .6803148591$ 
H 1.25714066170 .79705891480 .6804252893

H - $1.29233508680 .8851580961 \quad-0.0057781629$

3d propane, $\mathrm{HF}$

H $1.3439888197 \quad-0.91780935350 .8172864967$

C $1.272894395 \quad 0.0134253208 \quad 0.2604871821$

H $1.2925022810 .8294454808 \quad 0.9788217881$

C $-0.00063634220 .0538040651 \quad-0.5821885287$

$\begin{array}{llll}\text { H } 2.1602720309 & 0.0969840122 & -0.3604935065\end{array}$

C $-1.2723294667-0.06119198380 .256373433$

H $0.0241869882-0.7526845036-1.3119909846$

H $-0.02687950920 .9803779592-1.1517705618$

H -1.34203043210 .75221211130 .9745611028$

H $-1.2905439175-0.99504318550 .8130314679$

H $-2.1610677783-0.0297069181-0.3674783194$

3d propane, MP2

H $1.3276355806 \quad-0.9256093990 .8228236176$

$\begin{array}{llll}\text { C } & 1.2684018313 & 0.0131317579 & 0.2622340724\end{array}$

H $1.27575290420 .8351338271 \quad 0.9856031311$

C $-0.0006439743 \quad 0.054448226 \quad-0.5891578298$

$\begin{array}{llll}\text { H } & 2.1691258192 & 0.0965496929 & -0.3529882641\end{array}$

C $-1.2678330441-0.06122269940 .2581346685$

H $0.0244137272-0.7598561904-1.3222139618$

H $-0.02712747340 .989301782-1.1605056373$

H -1.32566348070 .75886856610 .9813982383$

H $-1.2737807948-1.00187468740 .8186190089$

H $-2.1699051537-0.0306572989-0.360001599$

3d propane, DFT

H $1.3480001116 \quad-0.92342012690 .8226711404$

C $1.27672524820 .0135057709 \quad 0.2608415877$

H $1.29620944860 .8342023913 \quad 0.9851620388$

C $-0.00063826980 .0539656499 \quad-0.5839361991$

H $2.1725238067 \quad 0.0973739426-0.36082146$

C $-1.2761595342-0.06133683990 .2567152793$

H $0.0243268761-0.7569782452-1.3206680825$

H $-0.02703783170 .9861892702-1.159513572$

H -1.34602873280 .75674024320 .9808912742$

H $-1.2942380697 \quad-1.00088228120 .818400443$

H $-2.1733202744-0.0300326788-0.3678457891$

3a propyl anion, $\mathrm{HF}$

H $1.2554617765 \quad-0.57790060341 .139148052$

C $1.1741638756 \quad 0.20601076450 .3868948285$

H 1.02640187261 .14695261770 .9158623795 
C $-0.0161693239-0.0752128526-0.564395312$

$\begin{array}{llll}\text { H } & 2.1331247992 & 0.2643429072 & -0.1462567258\end{array}$

C $-1.4102136295-0.18192655010 .0412785901$

H $0.223796592-0.9978585739-1.1042568434$

H $-0.00334037710 .7125621932-1.3256800803$

H $-1.67707312750 .7529196157 \quad 0.5561668684$

H $-1.4450570684-0.99424632710 .7823477099$

3a propyl anion, MP2

$\begin{array}{llll}\mathrm{H} & 1.252107 & -0.584861 & 1.136488\end{array}$

$\begin{array}{llll}\mathrm{C} & 1.180529 & 0.206030 & 0.380513\end{array}$

$\begin{array}{llll}\mathrm{H} & 1.021488 & 1.151945 & 0.911624\end{array}$

$\begin{array}{llll}\text { C } & -0.023760 & -0.076092 & -0.563457\end{array}$

$\begin{array}{llll}\mathrm{H} & 2.143447 & 0.263263 & -0.165000\end{array}$

$\begin{array}{llll}\mathrm{C} & -1.396573 & -0.178104 & 0.056850\end{array}$

$\mathrm{H} \quad \begin{array}{llll}\mathrm{H} & 0.216572 & -1.008689 & -1.103471\end{array}$

$\mathrm{H} \quad-0.013127 \quad 0.721027 \quad-1.327395$

$\begin{array}{llll}\mathrm{H} & -1.709151 & 0.764140 & 0.536828\end{array}$

$\begin{array}{llll}\mathrm{H} & -1.472513 & -1.017825 & 0.767490\end{array}$

3a propyl anion, DFT

H $1.2852746887 \quad-0.5808151898 \quad 1.1331275657$

C 1.19233449710 .20700200470 .3759143066

H $1.0546535662 \quad 1.15582364150 .9083118681$

$\begin{array}{llll}\text { C } & -0.031025199 & -0.0760427041 & -0.5555785945\end{array}$

H $2.1456709817 \quad 0.2603014363 \quad-0.1903162187$

$\begin{array}{llll}\text { C }-1.3905871155 & -0.175907595 & 0.0676494859\end{array}$

H $0.2082414373-1.0076587809-1.0993130698$

H $-0.02104736320 .7189575713-1.3228329236$

H -1.76740385760 .75644722590 .5057047325$

H $-1.529722549-1.03336613750 .737406858$

3ts inter propyl- + propane, ts, HF

H $1.3384087224-1.3979723267-0.8843017911$

$\begin{array}{llll}\text { C } 0.6956270439 & -1.2795687423 & 0.0001804576\end{array}$

H $1.3383748444-1.39801815390 .8846790024$

C $-0.3975227573-2.34327317740 .0001318191$

H 0. 0. 0.0001213308

C $0.0486046501-3.8147499614-0.0002830539$

H $-1.0448127144-2.1892155785-0.8685453215$

H $-1.0445014648-2.18961452860 .8691102957$ 
H $0.6566136042-4.03385937420 .8764411922$

H $0.6563186814-4.0334471073-0.8773143769$

H $-0.7998070372-4.5067361841-0.0003050027$

C $-0.69562704391 .2795687423 \quad 0.0001804576$

C 0.39752275732 .34327317740 .0001318191

H -1.33837484441 .39801815390 .8846790024$

H $-1.33840872241 .3979723267-0.8843017911$

C $-0.04860465013 .8147499614-0.0002830539$

H $1.0448127144 \quad 2.1892155785 \quad-0.8685453215$

H 1.04450146482 .18961452860 .8691102957

H $0.79980703724 .5067361841-0.0003050027$

H -0.65661360424 .03385937420 .8764411922$

H $-0.65631868144 .0334471073-0.8773143769$

3ts inter propyl-+ propane, ts, MP2

H $1.2506801933-1.3821667103-1.1078862911$

$\begin{array}{llll}\text { C } & 0.7481183819 & -1.2287816379 & -0.1373084207\end{array}$

H $1.5022593706-1.27988416640 .6663800747$

C $-0.3533790474-2.2495234157 \quad 0.0764617322$

H 0.0 .0 .1393468417

C $0.0367524533-3.73864922220 .0607013862$

H $-1.1318668977-2.0961860489-0.6901786283$

H $-0.8495556427-2.0403492487 \quad 1.0387427376$

H $0.7767703378-3.94599614110 .8423763062$

H $0.4941951857-4.0004516873-0.9005223271$

H $-0.8305840366-4.40164629350 .2216333627$

C $-0.7481183819 \quad 1.2287816379-0.1373084207$

C $0.35337904742 .2495234157 \quad 0.0764617322$

H $-1.5022593706 \quad 1.27988416640 .6663800747$

H $-1.25068019331 .3821667103-1.1078862911$

C $-0.0367524533 \quad 3.73864922220 .0607013862$

H $1.13186689772 .0961860489-0.6901786283$

H $0.84955564272 .0403492487 \quad 1.0387427376$

H 0.83058403664 .40164629350 .2216333627

H -0.77677033783 .94599614110 .8423763062$

H $-0.49419518574 .0004516873-0.9005223271$

3ts inter propyl- + propane, ts, DFT 
H $1.2133497397-1.3836088234-1.0771716238$

C $0.6793776342-1.2761098286-0.1196334879$

H $1.4128060548-1.29350253810 .7019603478$

C $-0.3944074883-2.33122165850 .0536328903$

H 0 . 0. -0.1216050548

C $0.0498710838-3.81020631750 .0643279896$

H $-1.147276595-2.2155983114-0.7440516326$

H $-0.9409216351 \quad-2.14051783360 .992137046$

H $0.7627274608-3.9946722060 .8762511672$

H $0.5543892914-4.0696919945-0.8735429031$

H $\quad-0.8018092629 \quad-4.50186112430 .195255774$

C $-0.6793776342 \quad 1.2761098286 \quad-0.1196334879$

C $0.3944074883 \quad 2.33122165850 .0536328903$

H $-1.4128060548 \quad 1.29350253810 .7019603478$

H $-1.2133497397 \quad 1.3836088234-1.0771716238$

C -0.04987108383 .81020631750 .0643279896$

H $1.1472765952 .2155983114-0.7440516326$

H 0.94092163512 .14051783360 .992137046

H 0.80180926294 .50186112430 .195255774

H -0.76272746083 .9946722060 .8762511672$

H $-0.55438929144 .0696919945-0.8735429031$

13ts intra propyl-, intramolecular $\mathrm{H}+$ ts, $\mathrm{HF}$ H $2.0746962245 \quad-0.55810817180 .0753648832$

C $1.1770150852 \quad 0.014226983 \quad 0.3439563079$

H $\quad-0.0000932242-0.28836105641 .1456555213$

$\begin{array}{llll}\text { C } & 0.0000421742 & -0.0773266321 & -0.6495916512\end{array}$

$\begin{array}{lllll}\mathrm{H} & 1.4720242693 & 1.0538224125 & 0.5288200834\end{array}$

C $-1.177062527 \quad 0.01433301490 .3437907597$

H $0.0000299417-1.0661120489-1.1144715258$

H $0.0001329798 \quad 0.6553188452-1.4679876169$

H $-2.0747599451 \quad-0.55791593420 .0750692205$

H $-1.471998641 .0539557594 \quad 0.5286169358$

13ts intra propyl-, intramolecular $\mathrm{H}+$ ts, MP2 H $2.0969103715 \quad-0.52715479830 .0689106667$

C $1.17350043440 .0176537423 \quad 0.3413869745$

H $-0.00009489-0.3158088632 \quad 1.1429173923$

$\begin{array}{llll}\text { C } & 0.0000419428 & -0.0876344349 & -0.653544889\end{array}$

H $1.4217039494 \quad 1.0688349607 \quad 0.5650718437$

C -1.17354682340 .01776173950 .3412218744$

H $0.0000268691-1.0938995657-1.0975148319$

H $0.00013404640 .6393379809-1.4872562656$

H $-2.0969685651 \quad-0.52696179050 .0686156205$

H - 1.42168509961 .06896579510 .5648718146 
13ts intra propyl-,intramolecular $\mathrm{H}+$ ts, DFT H $2.0646653796 \quad-0.6080966980 .1037455497$

C $1.1825990256 \quad 0.01389770710 .3418741694$

H $-0.0000942595 \quad-0.26089865941 .1692550547$

$\begin{array}{llll}\text { C } & 0.0000426843 & -0.0694323302 & -0.6521801057\end{array}$

H $1.51127028491 .0508454799 \quad 0.5185942955$

C -1.18264582980 .01400654480 .3417077803$

H $0.0000314257-1.0559994065-1.1374674209$

H $0.00013431670 .680239874-1.4643752683$

H $-2.0647359105-0.6079066820 .1034550566$

H $-1.511246517 \quad 1.05098456220 .518381669$

14 1-butyl anion, HF

H $0.6972902561 \quad 0.87711521492 .0604116307$

C 0.0703154310 .1 .9033607371

H $0.6972902561 \quad-0.87711521492 .0604116307$

C -0.53123647360 .0 .4921715789$

H $-0.6961786557 \quad 0.2 .6799861893$

C $0.5187045797 \quad 0 .-0.6446676057$

H $-1.1756731388 \quad 0.87156978350 .3740126918$

H $-1.1756731388-0.87156978350 .3740126918$

H $1.1642351818-0.8703604362-0.4751888972$

$\begin{array}{llll}\text { H } & 1.1642351818 & 0.8703604362 & -0.4751888972\end{array}$

C $0.02817460380 .-2.0910607451$

H $-0.5956373941-0.8874721173-2.2786404154$

H $-0.59563739410 .8874721173-2.2786404154$

14 1-butylanion, MP2

H $0.7149159705 \quad 0.8840877682 .0383636656$

C $0.0793462897 \quad 0.1 .9027763364$

H $0.7149159705-0.8840877682 .0383636656$

C $-0.5417079386 \quad 0.0 .4982193567$

H -0.67418181730 .2 .7015613722$

C $0.50888622640 .-0.6457662588$

H -1.18669091550 .87971225740 .3704315263$

H $-1.1866909155-0.87971225740 .3704315263$

H $1.1536263976-0.8800694985-0.464044736$

H $1.15362639760 .8800694985-0.464044736$

C $0.02777057330 .-2.0845079061$

H $-0.5676459961-0.9030916713-2.3076957267$

H $-0.56764599610 .9030916713 \quad-2.3076957267$ 

14 1-butyl anion, DFT
H 0.72668942780 .88244248792 .0500057105
C $0.0890867142 \quad 0.1 .9118401905$
H $0.7266894278-0.88244248792 .0500057105$
C -0.54682937720 .0 .5097945612$
$\mathrm{H}-0.655542233 \quad 0.2 .7175265385$
C $0.4969498047 \quad 0 . \quad-0.6551672751$
H -1.19685815330 .87674184580 .391004759$
H $-1.1968581533 \quad-0.87674184580 .391004759$
H $1.1454940234-0.8778905852-0.4748282654$
H $1.14549402340 .8778905852 \quad-0.4748282654$
C $0.02405451770 .-2.0872412459$
H $-0.5373391595-0.9051403703-2.3626241656$
H $-0.5373391595 \quad 0.9051403703 \quad-2.3626241656$

14ts intra 1-butyl anion, $\mathrm{H}+$ ts, $\mathrm{HF}$

H $2.1271325177 \quad-0.5524076512 \quad 1.0023808119$

C $1.332473603 \quad 0.12927181340 .6885575578$

H 0.0 .1 .2349806289

C $0.751839531-0.2134647356-0.6837576817$

H $1.7161574275 \quad 1.15601495790 .726301769$

C $-0.751839531 \quad 0.2134647356-0.6837576817$

H $0.7917962474-1.2975210551-0.8288425918$

H $1.263116108 \quad 0.2272322421-1.5461295597$

H $-1.263116108-0.2272322421-1.5461295597$

H $-0.7917962474 \quad 1.2975210551 \quad-0.8288425918$

C $-1.332473603 \quad-0.12927181340 .6885575578$

H $-2.1271325177 \quad 0.5524076512 \quad 1.0023808119$

H $-1.7161574275-1.15601495790 .726301769$

14ts intra 1-butylanion, $\mathrm{H}+$ ts, MP2

H $2.0895595809 \quad-0.55795647261 .0518568227$

C $1.3283460858 \quad 0.1417999061 \quad 0.6825245348$

H 0. 0. 1.2209136136

C $0.7582159228 \quad-0.2158257599 \quad-0.6846461204$

H $1.7111109183 \quad 1.17405435620 .7271442012$

C $-0.75821592280 .2158257599 \quad-0.6846461204$

H $0.7878412662-1.3097748986-0.8161663482$

H $1.2623154851 \quad 0.2222333124-1.5605619685$

H $-1.2623154851-0.2222333124-1.5605619685$

H $-0.7878412662 \quad 1.3097748986-0.8161663482$

C $-1.3283460858-0.14179990610 .6825245348$

H $-2.0895595809 \quad 0.5579564726 \quad 1.0518568227$

H $-1.7111109183-1.17405435620 .7271442012$

14ts intra 1-butyl anion, $\mathrm{H}+$ ts, DFT 
H $2.0810428133 \quad-0.59975606321 .0522236654$

C $1.3364506505 \quad 0.11923556880 .688850109$

H 0. 0. 1.2352040535

C $0.7703421236-0.1944525642-0.6929183685$

H $1.7314706105 \quad 1.14266169960 .7783084194$

C $-0.77034212360 .1944525642 \quad-0.6929183685$

H $0.8397181428-1.2776433501-0.877951542$

H $1.2665359745 \quad 0.293188826-1.5457730129$

H $-1.2665359745-0.293188826-1.5457730129$

H $-0.8397181428 \quad 1.2776433501-0.877951542$

$\begin{array}{llll}\text { C } & -1.3364506505 & -0.1192355688 & 0.688850109\end{array}$

H -2.08104281330 .59975606321 .0522236654$

H $-1.7314706105-1.14266169960 .7783084194$

15 1-pentyl anion, HF

H $0.8486481746 \quad 0.8870860462 .7926206495$

C 0.2018128965 0. 2.7123346522

H $0.8486481746 \quad-0.8870860462 .7926206495$

C -0.5215434056 0. 1.3667699183

H $-0.25502036590 .-3.3971895165$

C 0.32648853340 .0 .0715784602

H $-1.18586079370 .8703874644 \quad 1.3075210537$

H $-1.1858607937-0.8703874644 \quad 1.3075210537$

H $0.9825521941 \quad-0.8721305906 \quad 0.0826829115$

H $0.98255219410 .8721305906 \quad 0.0826829115$

C -0.4909724906 0. -1.2246190787

C $0.3596083926 \quad 0 .-2.4968705813$

H $-1.14709149320 .8709833088-1.2357701501$

H - $1.1470914932-0.8709833088-1.2357701501$

H $1.0030803221 \quad-0.8769338573-2.5360398183$

H $1.00308032210 .8769338573 \quad-2.5360398183$

15 1-pentylanion, MP2

H $0.8315108505 \quad 0.90229365742 .8235016782$

C 0.205030929 0. 2.7076663151

H $0.8315108505 \quad-0.90229365742 .8235016782$

C $-0.5124691467 \quad 0.1 .3701248974$

H -0.2649984076 o. -3.4092459005

C 0.3322506167 0. 0.0650657733

H $-1.1775077783 \quad 0.8803170562 \quad 1.3004192935$

H $-1.1775077783-0.8803170562 \quad 1.3004192935$

H $0.9906343685-0.88076123550 .0810952091$

H $0.99063436850 .8807612355 \quad 0.0810952091$

C -0.4953161769 0. -1.2242781283

$\begin{array}{llll}\text { C } & 0.3515051045 & 0 . & -2.5006419951\end{array}$

H $-1.15416637840 .8796353167-1.2233795022$ 
H $-1.1541663784-0.8796353167-1.2233795022$

H $0.9990241619-0.8835685363-2.5308243156$

H $0.99902416190 .8835685363 \quad-2.5308243156$

15 1-pentyl anion, DFT

H 0.7824060020 .9063669382 .9116817865

C $0.1943736671 \quad 0.2 .7132959984$

H $0.782406002-0.9063669382 .9116817865$

C -0.5002223569 0. 1.3788064509

H $-0.3134756891 \quad 0 .-3.4149569131$

$\begin{array}{llll}\text { C } & 0.3538367759 & 0.0 .0609092107\end{array}$

H -1.16653819470 .87850760961 .3030745387$

H $-1.1665381947-0.8785076096 \quad 1.3030745387$

H $1.013666583 \quad-0.87774317890 .0804316424$

H $1.013666583 \quad 0.87774317890 .0804316424$

C -0.4823476973 0. -1.2241910765

$\begin{array}{llll}\text { C } & 0.3323640187 & 0 . & -2.5237251125\end{array}$

H $-1.14661241070 .8756949144-1.2190193308$

H $-1.1466124107-0.8756949144-1.2190193308$

H $0.9798026425 \quad-0.881523808-2.5839765931$

H $0.97980264250 .881523808 \quad-2.5839765931$

15ts intra 1-pentyl-, $\mathrm{h}+$ ts, $\mathrm{HF}$

H $1.48572939432 .1486550161 \quad-0.6090167945$

C 1.02016852961 .40125176380 .0470384107

H $1.4857293943-2.1486550161-0.6090167945$

C $-0.4877308225 \quad 1.286544744 \quad-0.2365072265$

H $1.1843070941 .7292439681 \quad 1.0837110532$

C -1.08885468250 .0 .3561872293$

H $-0.6437570972 \quad 1.2518333427-1.3198135787$

H $-1.0842869372 .1418964082 \quad 0.1133472264$

H -0.9292935520 .1 .4378191378$

C $-0.4877308225-1.286544744-0.2365072265$

H -2.17385763750 .0 .2068020859$

C $1.0201685296-1.40125176380 .0470384107$

H $-0.6437570972-1.2518333427-1.3198135787$

H $-1.084286937-2.14189640820 .1133472264$

H $1.184307094-1.72924396811 .0837110532$

H $1.36304189140 .-0.0445746222$

15 ts intra 1-pentyl-, $\mathrm{h}+\mathrm{ts}, \mathrm{MP} 2$

H $1.50791745742 .1219104024-0.6183075503$

C $1.0135405901 \quad 1.396191643 \quad 0.0514397144$

H $1.5079174574-2.1219104024-0.6183075503$

C $-0.488699004 \quad 1.2813352662-0.2456834937$

H 1.17566482121 .71860837021 .095950793 
C -1.07954513160 .0 .3706095737$

H $-0.63398906141 .2091198876-1.337437393$

H - $-1.10603397262 .143717517 \quad 0.0781755604$

H -0.87566103150 .1 .4535359611$

C $-0.488699004-1.2813352662-0.2456834937$

H -2.17616788850 .0 .2517114231$

$\begin{array}{llll}\text { C } & 1.0135405901 & -1.396191643 & 0.0514397144\end{array}$

H $-0.6339890614-1.2091198876-1.337437393$

H $-1.1060339726-2.143717517 \quad 0.0781755604$

H $1.1756648212-1.71860837021 .095950793$

H 1.3438821873 0. -0.0347422955

15ts intra 1-pentyl-, $\mathrm{h}+$ ts, DFT

H $1.5212881322 .1080998043-0.6324230948$

C 1.01691649691 .40370166040 .0507020662

H $1.521288132-2.1080998043-0.6324230948$

C $-0.4884808101 \quad 1.2910814647-0.2374751069$

H $1.2030221594 \quad 1.7325997837 \quad 1.0878751332$

C -1.09200689010 .0 .3590166175$

H $-0.63829920151 .2476877292-1.3293503574$

H -1.1022070582 .15117042880 .1006542543$

$\mathrm{H}-0.9248893165$ 0. 1.4473052611

C $-0.4884808101-1.2910814647-0.2374751069$

H -2.1846069880 .0 .214467155$

C $1.0169164969-1.40370166040 .0507020662$

H $-0.6382992015-1.2476877292-1.3293503574$

H $-1.102207058-2.15117042880 .1006542543$

H $1.2030221594-1.73259978371 .0878751332$

H $1.3527013396 \quad 0 .-0.0281075031$

4d tetrafluoroethyl +, HF

H $0.79383843140 .3723280221-1.4711555157$

$\begin{array}{llll}\text { C } & 0.4469071865 & 0.5929000214 & -0.4663106453\end{array}$

F 1.43592633590 .80626901910 .3511160825

$\begin{array}{llll}\text { C } & -0.3027310259 & -0.6642902212 & 0.0678643012\end{array}$

$\begin{array}{llll}\text { F } & -0.4146697069 & 1.5672848608 & -0.4548673484\end{array}$

F $0.2852660187-1.5488582105 \quad 0.6531916239$

F $-1.4908443582-0.818471983-0.120347738$

4d tetrafluoroethyl +, MP2

H $0.8006504408 \quad 0.3730763191 \quad-1.4860856758$

C $0.44759005460 .5910431008-0.4696311576$

$\begin{array}{lllll}\text { F } & 1.459218545 & 0.8074662829 & 0.3610261187\end{array}$

C $-0.3019346913-0.66579179750 .0646181546$

F $-0.4312893042 \quad 1.5848947737 \quad-0.4623425803$

$\begin{array}{llll}\text { F } & 0.2990968435 & -1.5646025411 & 0.66284654\end{array}$ 


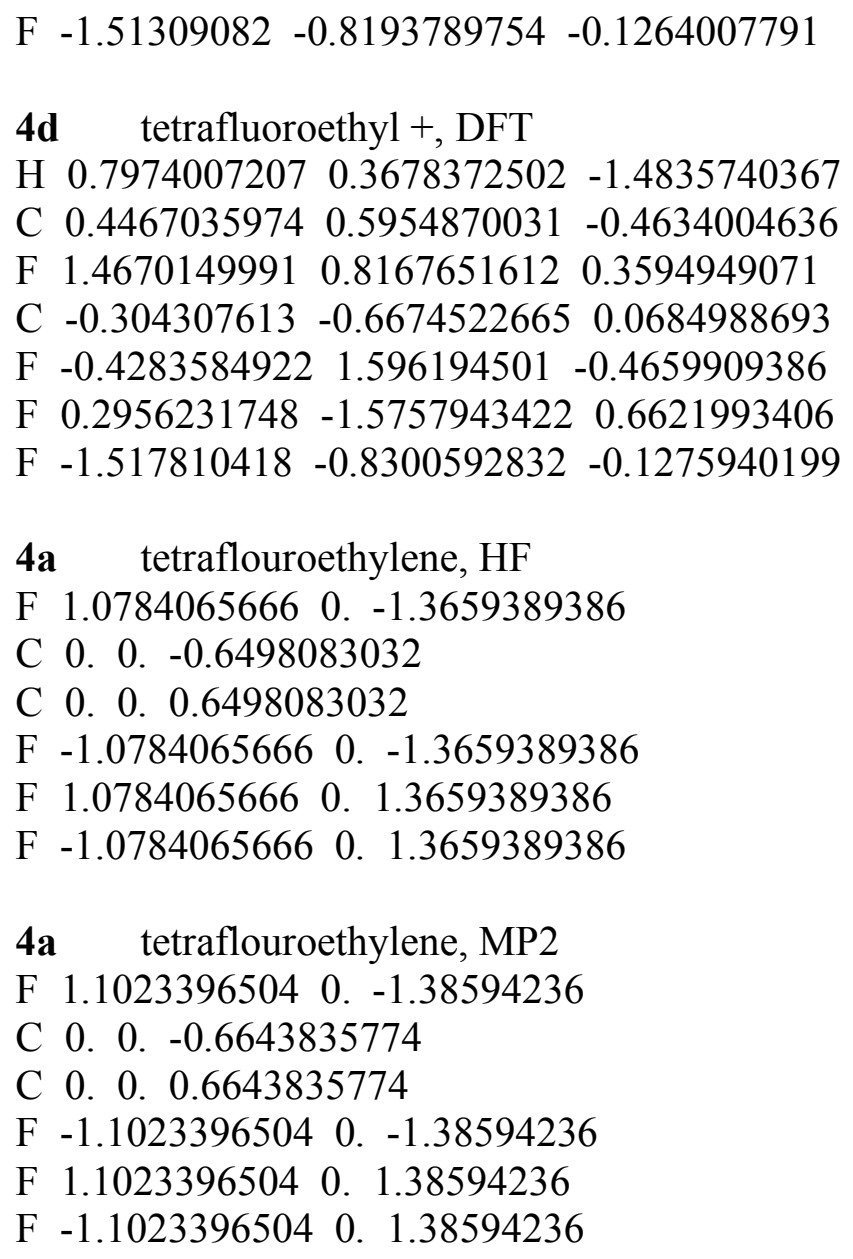

4a tetraflouroethylene, DFT

F $1.10246959720 .-1.3890655016$

C 0. 0. -0.6612386122

C 0. 0.0 .6612386122

F $-1.10246959720 .-1.3890655016$

F 1.10246959720 .1 .3890655016

F $-1.1024695972 \quad 0.1 .3890655016$

4ts tetrafluoroethyl + , proton transfer ts, HF H $0.0506561997 \quad-0.02218884290 .1126203973$ C $0.6938447373 \quad 1.2501796247 \quad 0.1619059381$

F $1.55090385 \quad 1.1465277715 \quad 1.1343884068$

C -0.42350662432 .05360480620 .3648470138$

F $1.25711128161 .290567165-1.015615874$

F $-0.8765312792 \quad 2.2706496962 \quad 1.5020787703$

F $-1.15015781252 .4140157366-0.57871544$

C $-0.5266401954-1.3231324537 \quad 0.2077364777$

F $-0.3784437457 \quad-1.6572364756 \quad 1.455725596$

C $0.2552133005-1.9800848885-0.7369014193$ 


$$
\begin{array}{llll}
\text { F } & -1.7673660412 & -1.0692244403 & -0.1111168471 \\
\text { F } & 0.0036826175 & -1.914897022 & -1.9537334183 \\
\text { F } & 1.3558985173 & -2.4783150636 & -0.4439165781
\end{array}
$$

4ts tetrafluoroethyl + , proton transfer ts, MP2 H $0.0677273456 \quad-0.02947208370 .1495394137$

C $0.6933290869 \quad 1.20535241640 .1936935673$

F $1.5157037933 \quad 1.0979447168 \quad 1.2166098301$

C -0.44018681662 .0227457540 .3297214433$

F $1.2945311517 \quad 1.1750208665 \quad-0.9865895408$

F $-0.9461269715 \quad 2.2719612211 \quad 1.470714$

F $-1.15164085332 .3382727458-0.6792801727$

C $-0.4966361404 \quad-1.2908831585 \quad 0.2407286022$

F $-0.2878985228-1.6320998468 \quad 1.4953999047$

C $0.2450860216-1.9378860867-0.7606435123$

F $-1.7545810112 \quad-0.9748061017 \quad-0.0296497068$

F $-0.0577346178-1.812145579-1.9920200691$

F $1.3791603367-2.4604259635 \quad-0.5141331363$

4ts tetrafluoroethyl + , proton transfer ts, DFT H $0.0423763134-0.01844226630 .0940337306$

C $0.640895521 \quad 1.2471502204 \quad 0.1302798744$

F $1.579008471 \quad 1.0967949807 \quad 1.0530599012$

C $-0.4406035457 \quad 2.1010588362 \quad 0.4375183317$

F $1.1252138365 \quad 1.3068097142-1.1067837927$

F $-0.8059278272 \quad 2.3097559388 \quad 1.6398922875$

F $-1.24191882892 .5151238937 \quad-0.4629391453$

C $-0.5152360515-1.30202294320 .1486674395$

F $-0.4559661514-1.58802048021 .4405442364$

C $0.3137427435-2.0455147486-0.7191809876$

F $-1.7472682627-1.0346010453-0.2744709457$

F $0.1409324882-2.0335001684-1.9818791509$

F $1.4020186836 \quad-2.5707612692 \quad-0.3160613548$

5d methanol, HF

H $0.5239531743 \quad 0.8849140337 \quad 1.0208205507$

C 0.0067203908 0. 0.6568034987

H $0.5239531743 \quad-0.8849140337 \quad 1.0208205507$

O -0.1050277555 0. -0.7389169926

$\mathrm{H}-0.9972153120 .1 .0595436337$

H 0.7492086629 0. -1.1306697865

5d methanol, MP2

H $-0.4519428517 \quad 1.0295380 .9377216308$

$\begin{array}{llll}\text { C } & -0.0140585247 & 0.665109 & 0.0012945301\end{array}$

H $-0.61558491561 .029538-0.8394220688$ 
$\begin{array}{llll}\text { O } & 0.1157333838 & -0.750387 & -0.010656904\end{array}$

H $0.9926017458 \quad 1.072335-0.0914002612$

H $-0.7665839262-1.1189710 .0705882005$

5d methanol, DFT

H 0.53207756930 .89301796361 .0359976072

C $0.0123619367 \quad 0.0 .6675599613$

H $0.5320775693 \quad-0.89301796361 .0359976072$

O -0.1130082485 0. -0.7510579042

$\mathrm{H}-1.00039572040 .1 .0711078998$

H $0.7661349497 \quad 0 .-1.1399996481$

5a methoxide anion, $\mathrm{HF}$

H $0.5082616803 \quad 0.88033505381 .0177924949$

C 0. 0. 0.5402091317

H $0.5082616803 \quad-0.8803350538 \quad 1.0177924949$

O $0.0 . \quad-0.7868290344$

H $-1.0165233606 \quad 0.1 .0177924949$

5a methoxide anion, MP2

H $0.1 .0227253963-1.035027$

C 0. 0. -0.546551

H $-0.8857061743-0.5113626981-1.035027$

O 0.0 .0 .798048

H $0.8857061743-0.5113626981-1.035027$

5d methoxide anion, DFT

H 0.5135645340 .88951986591 .0381441085

C 0.0 .0 .5390259717

H $0.513564534 \quad-0.88951986591 .0381441085$

O $0 . \quad 0 .-0.7935735194$

H -1.02712906810 .1 .0381441085$

5cx methanol + methoxide, complex, HF

H $1.05822919590 .5630368552-2.1018235254$

C $1.5007693712-0.1756648449-1.4283400945$

H $0.9947011644-1.1258698014-1.6182733386$

$\begin{array}{lllll}\text { O } & 1.4273988269 & 0.2054987189 & -0.1071649263\end{array}$

H $2.5487382841-0.2958379204-1.710508217$

H 0.46791014680 .29512804240 .1349344022

$\begin{array}{llllll}\text { O } & -1.0930987502 & 0.3975244518 & 0.3178170615\end{array}$

$\begin{array}{llll}\text { C } & -1.7825458144 & -0.2797786741 & 1.2484728298\end{array}$

H $-2.6913062671 \quad-0.78710596140 .8559966598$

H $-2.15618950220 .3496795472 \quad 2.0862931035$

H - $-1.2058249763-1.09055501371 .7473674217$ 
5cx methanol + methoxide, complex, MP2

H $1.6163526201-1.1173575518 \quad 1.0744561963$

$\begin{array}{lllll}\text { C } & 1.7998025234 & -0.2596603263 & 0.4009242989\end{array}$

H $1.5857949073 \quad 0.6503836746 \quad 0.994964858$

O $1.0665039143-0.3282597603-0.7771117727$

H $2.8801368289-0.24849170550 .1782446308$

H $0.0133138928-0.3985728202-0.4652961849$

O $-1.2360110543-0.44215805630 .0046911801$

C $-1.6787686556 \quad 0.84276303380 .2002380208$

H $-2.0126132647 \quad 1.0454728144 \quad 1.2454208053$

H $-2.5502748492 \quad 1.1202692494-0.4388670593$

H $-0.90285992611 .6130245618-0.0165290263$

5cx methanol + methoxide, complex, DFT

H $1.16979097790 .4373983125-2.1586375457$

$\begin{array}{llll}\text { C } & 1.4644358218 & -0.2675044918 & -1.3546611977\end{array}$

H $0.8684478866-1.1868347269-1.5183080941$

$\begin{array}{lllll}\text { O } & 1.3159143441 & 0.2600343886 & -0.0789135988\end{array}$

H $2.5206271106-0.53323069-1.5365575248$

H 0.25071051250 .40786713490 .1145340872

O $-1.0993999475 \quad 0.55285170240 .3027413027$

C $-1.6422895445-0.3476678291 .1743582646$

H $-2.5305270797 \quad-0.89284620780 .7634902704$

H -2.009636884 0.10701082342 .1309884584

H $-0.9344053605 \quad-1.15141944931 .4956863167$

5ts $\mathrm{MeOH}+\mathrm{MeO}-$, ts, $\mathrm{HF}$

H $0.6772850821 .6544391656-1.2320535306$

$\begin{array}{llll}\text { C } & 0.2706308652 & 2.0006407988 & -0.2679118759\end{array}$

H 1.14479327922 .31142334590 .3296868942

$\begin{array}{lllll}\text { O } & -0.5096743774 & 1.0763246667 & 0.3515382808\end{array}$

H $-0.2884248998 \quad 2.9230830503 \quad-0.486446481$

H 0.0 .0 .3679562534

$\begin{array}{lllll}\text { O } & 0.5096743774 & -1.0763246667 & 0.3515382808\end{array}$

C $-0.2706308652-2.0006407988-0.2679118759$

H $0.2884248998-2.9230830503-0.486446481$

H $-0.677285082-1.6544391656-1.2320535306$

H $-1.1447932792 \quad-2.31142334590 .3296868942$

5ts $\mathrm{MeOH}+\mathrm{MeO}-$, ts, MP2

H $1.5490919807 \quad-0.0865850394-0.881245$

C $1.5011474960 .9370885741-0.457674$

H $2.5408032216 \quad 1.2004243585 \quad-0.170202$

O $0.6161620142 \quad 1.02963624860 .59823$

H $1.24481338081 .6093786069-1.306522$

H 0.0 .0 .636347 


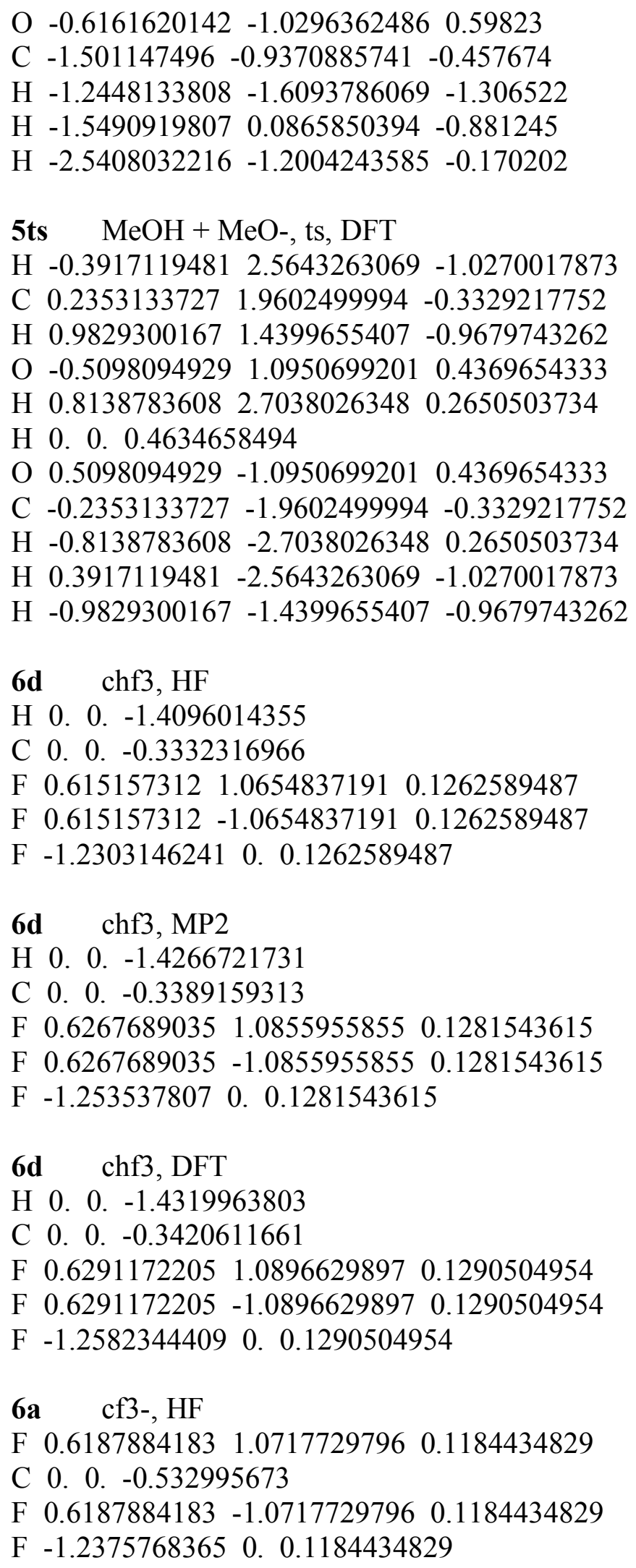


6a cf3-, MP2

F $0.6327064009 \quad 1.0958796326 \quad 0.121665213$

C 0. 0. -0.5474934585

F $0.6327064009-1.09587963260 .121665213$

F $-1.2654128018 \quad 0.0 .121665213$

6a cf3-, DFT

F $0.636109443 \quad 1.10177387440 .1232247972$

C 0. 0. -0.5545115874

F $0.636109443-1.10177387440 .1232247972$

F $-1.272218886 \quad 0.0 .1232247972$

6ts $\quad$ chf3 + ch3-, proton transfer ts, HF

F $-0.3308421958 \quad 1.19029597381 .9932201444$

C $-0.0019161718 \quad 0.0009005519 \quad 1.4198702373$

F $1.1911176892-0.3090553874 \quad 1.9962236177$

H -0.00000156350 .00000157890 .0000002435$

F $-0.8683420856-0.8774356925 \quad 1.9938137661$

C $0.0019168355-0.0009005808-1.4198701841$

F $0.3306768884-1.1903430913-1.9932189492$

F $0.86846276110 .8773161236-1.9938135359$

F $-1.19107332620 .3092219176-1.9962251057$

6ts chf3 + cf3-, proton transfer ts, MP2

F $-0.3373476145 \quad 1.21400978182 .0055551172$

C $-0.00191363150 .0009022248 \quad 1.4193101562$

F $1.2148733455-0.315280942 .0086205196$

H $0.0000000582 \quad-0.0000000568 \quad 0.0000000173$

F $-0.8856419977 \quad-0.89490038492 .0061580714$

C $0.0019137095-0.0009022759-1.4193101502$

F $0.3372780832-1.2140289761 \quad-2.0055554061$

F $0.88569332520 .8948497852 \quad-2.0061579636$

F $-1.2148552002 \quad 0.3153507744 \quad-2.0086203444$

6ts chf3 + cf3-, proton transfer ts, DFT

F $-0.3386735132 \quad 1.21922037692 .0229329317$

C $-0.0020279102 \quad 0.0015345521 \quad 1.4312994842$

F $1.2199131159 \quad-0.3163019317 \quad 2.0245914359$

H $-0.0000005313 \quad 0.0000011132-0.0000000018$

F $-0.889480922 \quad-0.89853480482 .0218738514$

C $0.0020274798 \quad-0.0015336429-1.4312994745$

F $0.3386126104-1.2192375306-2.0229302618$

F $0.8895260208 \quad 0.8984902666 \quad-2.0218747299$

F $-1.2198969658 \quad 0.3163628938 \quad-2.0245932338$

7d chloroform, HF 
H $0.00000051650 . \quad-1.5224496848$

C $0.00000019660 .-0.4512291612$

Cl $1.6813971992 \quad 0.0 .0829384243$

Cl $-0.8406986495 \quad-1.45613270390 .0829373952$

$\mathrm{Cl} \quad-0.8406986494 \quad 1.45613270390 .0829373952$

7d chloroform, MP2

H $0.00000060320 .-1.5344382087$

$\begin{array}{llll}\text { C } & 0.0000001758 & 0 . & -0.449338424\end{array}$

$\begin{array}{llllll}\mathrm{Cl} & 1.6832714944 & 0.0 .0829510171\end{array}$

Cl $-0.841635796-1.45775592440 .082950043$

Cl $-0.841635796 \quad 1.4577559244 \quad 0.082950043$

7d chloroform, DFT

H $-0.00004411330 .-1.5356764256$

C 0.000077885 0. -0.4534033248

$\mathrm{Cl} 1.7038337317 \quad 0.0 .0834672179$

Cl $-0.8519293128-1.47560751920 .0834456962$

$\mathrm{Cl} \quad-0.8519293128 \quad 1.4756075192 \quad 0.0834456962$

7a $\quad \mathrm{CCl} 3-, \mathrm{HF}$

Cl $-0.8524759873 \quad 1.4753973907 \quad 0.0796823604$

C $0.00309356240 .-0.7041026518$

Cl $1.7038601291 \quad 0.0 .0891420975$

Cl $-0.8524759873 \quad-1.4753973907 \quad 0.0796823604$

$7 \mathbf{a}$ CCl3-, MP2

Cl $-0.8526511706 \quad 1.47626003830 .0789199993$

C 0.0020490157 0. -0.6970485035

$\begin{array}{llllll}\mathrm{Cl} & 1.7045791591 & 0 . & 0.0881771202\end{array}$

Cl $-0.8526511706-1.4762600383 \quad 0.0789199993$

7a $\quad \mathrm{CCl} 3-, \mathrm{DFT}$

Cl $-0.8763345407 \quad 1.5183552833 \quad 0.0829291416$

C $0.00382369260 .-0.7372060115$

$\mathrm{Cl} 1.7513195427 \quad 0.0 .0943320737$

Cl $-0.8763345406-1.51835528340 .0829291416$

7ts $\mathrm{CHCl} 3+\mathrm{CCl} 3-$, proton transfer ts, $\mathrm{HF}$

H 0. 0. 0.0000000191

C 0. 0. 1.3956274493

$\mathrm{Cl}-1.6698693202 \quad 0.2155885197 \quad 2.0417954631$

Cl $1.0216397949 \quad 1.3383549925 \quad 2.0417954631$

Cl $0.6482295253-1.55394351212 .0417954631$

C 0. 0. -1.3956274399

Cl $-1.0213212355 \quad-1.338598099-2.0417954646$ 
Cl $1.6699205769-0.2151910858-2.0417954646$

Cl $-0.6485993415 \quad 1.5537891848-2.0417954646$

7ts $\mathrm{CHCl} 3+\mathrm{CCl} 3-$, proton transfer ts, $\mathrm{MP} 2$ H 0.0 .0 . 0.

C 0. 0. 1.3822297452

Cl $-1.6730350138 \quad 0.21595456252 .0151218691$

$\begin{array}{lllll}\mathrm{Cl} & 1.0235396441 & 1.3409135421 & 2.0151218691\end{array}$

Cl $0.6494953697-1.55686810462 .0151218691$

C 0. 0. -1.3822297452

Cl $-1.0232877571-1.3411057739-2.0151218691$

Cl $1.6730755479-0.2156403061-2.0151218691$

Cl $-0.6497877908 \quad 1.5567460799-2.0151218691$

7ts $\mathrm{CHCl} 3+\mathrm{CCl} 3-$, proton transfer ts, $\mathrm{DFT}$

H 0. 0.0 .

C 0. 0. 1.3975897642

Cl $-1.6965364259 \quad 0.23017139782 .0635333873$

Cl $1.0476024907 \quad 1.3541579444 \quad 2.0635333873$

Cl $0.6489339352-1.5843293422 \quad 2.0635333873$

C 0. 0. -1.3975897642

Cl $-1.0297128918-1.3678106877-2.0635333873$

Cl $1.699415249-0.2078521791-2.0635333873$

Cl $\quad-0.6697023572 \quad 1.57566286667-2.0635333873$

8d acetaldehyde, $\mathrm{HF}$

H $0.1265239441 .1166922197-1.5523484724$

C $0.2690699808 \quad 1.0345216652 \quad-0.4777763481$

H $1.32533104681 .0130375253-0.2445314412$

C $-0.4103656356 \quad-0.2091270790 .020865141$

H $-0.1951639181 \quad 1.9057522783-0.0225332453$

$\begin{array}{llll}\text { O } & 0.1350420566 & -1.0901889823 & 0.5921063228\end{array}$

H $-1.4892535961-0.2663376818-0.1759701808$

8d acetaldehyde, MP2

H $-0.7991272409-0.5887447136 \quad-1.6572241837$

C $-0.90337494960 .0287837775-0.7588908294$

H $-0.81383043051 .0848383255-1.0153353238$

C $0.1515821079-0.36554113760 .2396934345$

H $-1.8933762916-0.17535577-0.3376144291$

$\begin{array}{lllll}\text { O } & 0.9857036396 & 0.3911910062 & 0.6963240834\end{array}$

H $0.1314633799-1.4297217367 \quad 0.5547682461$

8d acetaldehyde, DFT

H $-0.8020191819-0.5877637767-1.6560976843$

C $-0.90088123840 .0311534218-0.7574447243$ 
H $-0.8168041791 \quad 1.086077853-1.0179399546$

$\begin{array}{llll}\text { C } & 0.1534766148 & -0.3617053768 & 0.2402945969\end{array}$

H $-1.8928783762-0.1759343728-0.3409164997$

$\begin{array}{llllll}\text { O } & 0.9829676084 & 0.3861279395 & 0.695317065\end{array}$

H $0.132388612 \quad-1.42809148940 .5553183833$

8a acetaldehyde anion, HF

H $1.2456706540 .-1.3360846129$

$\begin{array}{llll}\text { C } & 0.1788654254 & 0 . & -1.1705306337\end{array}$

C -0.34950000020 .0 .0995032894$

H -0.4779253334 0. -2.0260200654

O $0.2145460621 \quad 0.1 .2102159069$

H -1.4603063683 0. 0.106541489

8a acetaldehyde anion, MP2

H $-0.3293861816-1.346813-1.2107921973$

$\begin{array}{llll}\text { C } & -0.0468358298 & -1.18553 & -0.1721640447\end{array}$

C 0.09390896120 .0973220 .3452003875

H $0.1279914047 \quad-2.045186 \quad 0.4704841998$

$\begin{array}{lllll}\text { O } & -0.0587851733 & 1.227763 & -0.2160886919\end{array}$

H $0.38923789960 .099146 \quad 1.4308014059$
8a acetaldehyde anion, DFT
H $-0.3288002248-1.3471807573-1.2086382758$
C $-0.0465853607-1.1816287676-0.1712433442$
$\begin{array}{llll}\text { C } & 0.0929076173 & 0.0959925553 & 0.3415195425\end{array}$
H $0.1271112061 \quad-2.04357389070 .4672486739$
$\begin{array}{lllll}\text { O } & -0.0581188337 & 1.2254044528 & -0.2136392913\end{array}$
H $0.3887061493 \quad 0.101336299 \quad 1.4288467422$

8ts acetaldehyde proton transfer ts, HF

H $0.5461847241-0.1136062349-0.0860754356$

C $0.8661179849-0.1880743881 \quad 1.3229813692$

H $1.7784917911-0.77353899951 .3328818406$

C $-0.2557378848-0.8044421503 \quad 1.9601386539$

H $1.0072489597 \quad 0.8552486891 .5818894283$

O $-0.4434550459-1.9822761022 \quad 2.1795631981$

H $-1.0745250686-0.10275865622 .2046849884$

$\begin{array}{llll}\text { C } & 0.43852317 & -0.0832905627 & -1.5285849986\end{array}$

H $1.45965793520 .1000048539-1.8431948748$

C $-0.48823813850 .9591889327-1.8428924776$

H $0.0789064832-1.0811686858-1.753060983$

$\begin{array}{llll}\text { O } & -0.2580306769 & 2.1281849093 & -2.0690132674\end{array}$

H - $1.54806983220 .6482575868-1.7913796909$

8ys acetaldehyde proton transfer ts, MP2 


$\begin{array}{lrrr}\mathrm{H} & 0.000000 & 0.000004 & 0.465453 \\ \mathrm{C} & 1.146570 & -0.827047 & 0.575811 \\ \mathrm{H} & 1.428343 & -0.675993 & 1.619357 \\ \mathrm{C} & 2.070225 & -0.321531 & -0.391584 \\ \mathrm{H} & 0.729687 & -1.809129 & 0.346213 \\ \mathrm{O} & 2.927284 & 0.569434 & -0.234773 \\ \mathrm{H} & 1.907358 & -0.716222 & -1.425455 \\ \mathrm{C} & -1.146572 & 0.827053 & 0.575806 \\ \mathrm{H} & -1.428344 & 0.676006 & 1.619352 \\ \mathrm{C} & -2.070225 & 0.321529 & -0.391586 \\ \mathrm{H} & -0.729691 & 1.809136 & 0.346201 \\ \mathrm{O} & -2.927282 & -0.569439 & -0.234770 \\ \mathrm{H} & -1.907360 & 0.716213 & -1.425460\end{array}$

8ts acetaldehyde proton transfer ts, DFT

$\begin{array}{llll}\mathrm{H} & 0.000000 & 0.000000 & 0.252604\end{array}$

$\begin{array}{llll}\text { C } & 0.761575 & 1.207291 & 0.357934\end{array}$

$\begin{array}{llll}\mathrm{H} & 0.781272 & 1.344069 & 1.439778\end{array}$

C $\quad 0.091439 \quad 2.225004 \quad-0.384882$

$\mathrm{H} \quad 1.703206 \quad 0.861231 \quad-0.070111$

$\begin{array}{llll}\mathrm{O} & -0.761575 & 3.031130 & 0.018296\end{array}$

$\mathrm{H} \quad 0.322847 \quad 2.215809 \quad-1.480650$

$\begin{array}{llll}\text { C } & -0.761575 & -1.207291 & 0.357934\end{array}$

$\mathrm{H} \quad-0.781272-1.344069 \quad 1.439778$

$\begin{array}{llll}\text { C } & -0.091439 & -2.225004 & -0.384882\end{array}$

$\mathrm{H} \quad-1.703206 \quad-0.861231-0.070111$

O $\quad 0.761575 \quad-3.031130 \quad 0.018296$

$\mathrm{H} \quad-0.322847 \quad-2.215809 \quad-1.480650$

9d acetonitrile, $\mathrm{HF}$

H 0.50941798910 .88233783941 .5393974094

C 0. 0. 1.1756263708

H $0.5094179891 \quad-0.88233783941 .5393974094$

C $0.0 . \quad-0.2895938146$

$\mathrm{H}-1.0188359781 \quad 0.1 .5393974094$

N 0. $0 .-1.4191982237$

9d acetonitrile, MP2

H $0.51319924840 .8888871727 \quad 1.5584600197$

C 0. 0. 1.1871438699

H $0.5131992484-0.8888871727 \quad 1.5584600197$

C 0. 0. -0.2755976703

H -1.02639849690 .1 .5584600197$

N 0. 0. -1.4492367509

9d acetonitrile, DFT 
H 0.51248091690 .8876429861 .5524225994

C 0. 0. 1.1764227936

H $0.5124809169-0.8876429861 .5524225994$

C 0. 0. -0.2805800119

H -1.02496183380 .1 .5524225994$

N 0. $0 .-1.4331892126$

9a acetonitrile anion, $\mathrm{HF}$

N $0.07287478860 .-1.3464886936$

C -0.1244458849 0. 1.1941443703

H 0.10056178020 .91922035911 .7089174114

$\begin{array}{llll}\text { C } & 0.0059047047 & 0 . & -0.1928800315\end{array}$

H $0.1005617802-0.91922035911 .7089174114$

9a acetonitrile anion, MP2

$\begin{array}{llll}\text { N } & 0.0922432885 & -1.373489 & 0.0073795096\end{array}$

C $-0.16554409711 .211418-0.0132436113$

H $0.2036634516 \quad 1.716413 \quad-0.9048363991$

$\begin{array}{llll}\text { C } & 0.0144458464 & -0.181151 & 0.001155675\end{array}$

H $0.0572190454 \quad 1.716413 \quad 0.9257071305$

9a acetonitrile anion, DFT

N $0.0428613959-1.36512678790 .0034289333$

C $-0.0758351109 \quad 1.1961224495 \quad-0.0060668471$

H $0.13635946341 .7393326105 \quad-0.9197469081$

C $0.0050368126-0.1832520670 .0004029475$

H $-0.0115994446 \quad 1.7393326105 \quad 0.9297277726$

9ts acetonitrile, proton transfer ts, $\mathrm{HF}$

H $-0.66761643521 .9110663123 \quad 0.3265651026$

C $-0.68392746991 .1718912918-0.4693038753$

H 0.00040153890 .00039078540 .0003938604

C $-0.0453423872 \quad 1.6978742026-1.6473241717$

H $-1.7099096190 .8935298053-0.6927421885$

N $0.5084208206 \quad 2.0615604543 \quad-2.5764942256$

C $0.6847771766-1.1710636597 \quad 0.4701353749$

H $1.7103761062-0.89233696030 .6948829547$

H $0.6697120536-1.9097599793-0.3262000797$

C $0.0451574649-1.6980574554 \quad 1.6471420932$

N $-0.5094140137-2.0625256312 \quad 2.5755233433$

9ts acetonitrile, proton transfer ts, MP2

H -1.33473456250 .60654500321 .4089566436$

C $-1.2277769742-0.20555092590 .6835801054$

H $-0.000007263 \quad 0.00007073470 .0000083574$

C $-2.3008492419-0.2024440665-0.2631508326$ 


\begin{tabular}{lrrrr} 
H & -1.1545297573 & -1.1679533157 & 1.19887 \\
N & -3.1041697338 & -0.1809433579 & -1.134462 \\
C & 1.227762296 & 0.2056941215 & -0.683563294 \\
H & 1.1545671619 & 1.1681573761 & -1.1987505 \\
H & 1.3346555406 & -0.6063302938 & -1.4090291 \\
C & 2.300851911 & 0.2024184349 & 0.263147531 \\
N & 3.1041864498 & 0.1807732748 & 1.13444209 \\
\multicolumn{4}{c}{} \\
9ts & acetonitrile, proton transfer ts, DFT \\
H & -1.007950 & -1.496867 & 0.897013 \\
C & -1.127380 & -0.883435 & 0.000345 \\
H & 0.000000 & 0.000001 & 0.000325 \\
C & -2.358299 & -0.172906 & -0.000110 \\
H & -1.007547 & -1.497391 & -0.895909 \\
N & -3.317368 & 0.492639 & -0.000381 \\
C & 1.127381 & 0.883436 & 0.000324 \\
H & 1.007887 & 1.496953 & 0.896925 \\
H & 1.007613 & 1.497307 & -0.895997 \\
C & 2.358299 & 0.172906 & -0.000020 \\
N & 3.317367 & -0.492640 & -0.000417
\end{tabular}

10d nitromethane, $\mathrm{HF}$

C $-0.0128860047 \quad 0.0003013945-1.3070610742$

$\begin{array}{lllll}\mathrm{N} & -0.0088966705 & 0.0003706324 & 0.1767056787\end{array}$

O $0.0464259787 \quad 1.0539284498 \quad 0.7180753911$

O $-0.0300689792-1.0543316537 \quad 0.7182769914$

H $1.023291928-0.0390677479-1.6136716035$

H $-0.47328973360 .9168674652-1.6360323862$

H $-0.5412654684-0.8789768797-1.6356883759$

10d nitromethane, MP2

C $-1.3221206818 \quad 0.0477153901 \quad-0.0015633327$

N $0.1695915004-0.0066247496-0.0129333495$

O $0.6915738582-1.11942139740 .0436349883$

O $0.77047948991 .0668531202-0.0363078616$

H $-1.63054263120 .0988766742 \quad 1.0429225771$

H $-1.692845051-0.8627941268-0.4662853645$

H $-1.62745956090 .9445437217 \quad-0.5353427568$

10d nitromethane, DFT

C -1.3274616019 $0.0477896503 \quad-0.0046111987$

N $0.1745122394 \quad-0.0067037322 \quad-0.0102447201$

O $0.692590196-1.1109656595 \quad 0.0433170205$

O $0.77089146631 .058349574-0.0360407488$

$\mathrm{H}-1.63228560580 .09884313611 .0407499553$

H $-1.6989048785-0.8630668049-0.4652516254$ 
H $-1.63347887820 .9453405761-0.5343282706$

10a nitromethane anion, $\mathrm{HF}$

H $-0.0752571499-0.9331396937-1.7698040582$

$\begin{array}{llll}\text { C } & 0.1041568013 & -0.0094934897 & -1.2622697738\end{array}$

N -0.00369302980 .00035171720 .0434947866$

H $0.36789025950 .9061015004-1.7470770029$

O $0.1972693227 \quad 1.0551246635 \quad 0.6870992224$

O $-0.3087346614-1.0449325246 \quad 0.6611553024$

10a nitromethane anion, MP2

H - $1.51726266881 .3461440714-0.1393450076$

C $-1.26819955690 .3020242559 \quad-0.0289092349$

N $0.0499307908 \quad-0.01197716890 .0011284773$

H $-1.9581076586-0.4650345340 .2877943874$

O $0.4159087808 \quad-1.21087442490 .2483194166$

O $0.9259731845 \quad 0.8846980863 \quad-0.2461800526$

10a nitromethane anion, DFT

H $-1.5244044697 \quad 1.3369887773 \quad-0.1733542929$

C -1.25650953590 .31275036890 .0290594193$

N $0.0434973501 \quad-0.0108308409 \quad-0.0010275598$

H $-1.9640599112-0.46901819590 .2527076239$

O $0.4146034017 \quad-1.2166771866 \quad 0.2322791844$

O $0.9257766165 \quad 0.883095073 \quad-0.2630938004$

10ts nitromethane proton transfer ts, HF

H $-0.0000000221-0.000243153-0.0000067696$

C $1.08880095090 .0158244211-0.9169671248$

H $1.0474934747-0.8809198726-1.513641907$

N $2.259336863-0.0055965756-0.1130819412$

H $1.0537885940 .9377571297-1.4743629805$

$\begin{array}{lllll}\text { O } & 2.7191175637 & 1.0394492152 & 0.2751475934\end{array}$

$\begin{array}{lllll}\text { O } & 2.7117572238 & -1.0695701552 & 0.2296422973\end{array}$

C $-1.0888009487-0.01636720860 .9169522798$

H $-1.0539954666 \quad-0.93853293081 .4739768482$

$\begin{array}{llll}\mathrm{N} & -2.259336861 & 0.005630592 & 0.1130828941\end{array}$

H $-1.04728327 \quad 0.88012587641 .5139886235$

O $-2.7192987697-1.0391365601-0.2756088002$

O $\quad-2.7115764351 \quad 1.0698614452 \quad-0.2291650173$

10ts nitromethane proton transfer ts, MP2

H $0.00001716320 .0004614575 \quad-0.0000186916$

C -1.05161356220 .00102290230 .9105849099$

H $-0.9873253979-0.9232713871 \quad 1.4781070715$

N -2.1859783329 0.000816350 .0672521013 

H $-0.9870018897 \quad 0.9258506847 \quad 1.4771986748$
O $-2.6254594559 \quad 1.0971451841 \quad-0.3514327187$
O $-2.6259474941-1.0957522152-0.3502782325$
C $1.05155091460 .0000126734-0.9105435553$
H $0.9865674501 \quad-0.9243333579-1.4779068269$
N $2.1860010003-0.0008934874-0.0672503676$
H $0.98765215090 .9247664499-1.4773654999$
O $2.6251587602-1.0977167505 \quad 0.3504445456$
O $2.6262872764 \quad 1.0951801063 \quad 0.3512322872$

10ts nitromethane proton transfer ts, DFT H $0.0000003910 .0000225565-0.000000308$ C $-1.0977857204 \quad 0.00055525560 .8748459284$

H - $-1.0059240043 \quad-0.92214120261 .4374335567$

$\mathrm{N}-2.25401566750 .00087444840 .067618724$

H $-1.00523575340 .9233410818 \quad 1.4371740511$

O $-2.7197493698 \quad 1.0947028606-0.3222357737$

O $-2.720564118-1.09271505-0.3219318516$

C $1.0977870951-0.0004647364-0.8748474978$

H $1.0052109823 \quad-0.9232007399-1.4372530275$

N $2.2540155862-0.0008803461-0.0676186958$

H $1.00594957330 .9222818431 \quad-1.4373566628$

O $2.7197173843 \quad-1.0947532488 \quad 0.3221491227$

O $2.7205949949 \quad 1.092664767 \quad 0.3220199539$

11d propyne, $\mathrm{HF}$

H $0.1 .0155310113-1.6178325843$

C 0. 0. -1.2399196481

H $-0.8794756541 \quad-0.5077655056-1.6178325843$

C 0. 0. 0.2262279104

H $0.8794756541 \quad-0.5077655057-1.6178325843$

C 0. 0. 1.4115781903

H 0.0 .2 .4661790376

11d propyne, MP2

H $0.1 .0232423757-1.632995$

C 0. 0. -1.248667

H $-0.8861538916 \quad-0.5116211878-1.632995$

C 0. 0. 0.215158

H $0.8861538916-0.5116211878-1.632995$

C 0. 0. 1.433639

H 0.0 .2 .498205

11d propyne, DFT

H $0.1 .0214704205-1.6288497448$

C 0. 0. -1.2388362311 
H $-0.8846193334-0.5107352103-1.6288497448$

C 0. 0. 0.2186262406

H $0.8846193334-0.5107352103-1.6288497448$

C 0. 0. 1.420797443

H 0. 0.2 .4830245194

11a methylacetylide, $\mathrm{HF}$

C 0. 0. 0.3395625302

C 0. 0. -1.1332532044

H $0.1 .011479041-1.54194396$

H $-0.8759665449-0.5057395205-1.54194396$

H $0.8759665449-0.5057395205-1.54194396$

C 0. 0. 1.5646626542

11a methylacetylide, MP2

C 0. 0. 0.331424

C 0. 0. -1.143415

H $0.1 .0191076387-1.558451$

H $-0.8825731043-0.5095538193-1.558451$

H $0.8825731043-0.5095538193-1.558451$

C 0. 0. 1.591217

11a methylacetylide, DFT

C 0. 0.0 .3346854038

C 0. 0. -1.1327846722

H $0.1 .0172790935-1.5569094333$

H $-0.8809895377-0.5086395468-1.5569094333$

H $0.8809895377-0.5086395468-1.5569094333$

C 0. 0. 1.576553985

11ts $\mathrm{MeCC}-+\mathrm{HCCMe}$, ts, $\mathrm{HF}$

H $-0.49938592640 .8828037024 \quad 4.4838421859$

C $-0.0076505735-0.0029412708 \quad 4.0858934148$

H $1.0044228005 \quad-0.02103461424 .4860073433$

C $-0.0048976777-0.00188292512 .6156743036$

H $-0.5302255817-0.87145290594 .4825216283$

C $-0.0026339551 \quad-0.00101264541 .4067022968$

H 0.0 .0 .

$\begin{array}{llll}\text { C } & 0.0026339657 & 0.0010126422 & -1.4067023341\end{array}$

C $0.0048976830 .0018829216-2.6156742886$

$\begin{array}{llll}\text { C } & 0.0076505643 & 0.0029412744 & -4.0858933989\end{array}$

H $0.5495711655 \quad 0.8595281571 \quad-4.482493977$

H $-1.00376850960 .0437507887-4.485989742$

H $0.479386011-0.8935951094-4.4838873996$

11ts MeCC- + HCCMe, ts, MP2 
H -0.00162407770 .9513006284 .5600598207$

$\begin{array}{llll}\text { C } & 0.0516049671 & -0.0475703038 & 4.10786006\end{array}$

H $0.9803363212 \quad-0.5140479093 \quad 4.4614931803$

C $-0.000063403 \quad 0.0182599392 .6403642802$

H $\quad-0.7813819591 \quad-0.633499143 \quad 4.5177490127$

C $-0.0485387707 \quad 0.12254697051 .4003759455$

H $-0.10395695670 .0620739612-0.0001269364$

C $-0.1286050988-0.0167540425-1.400587419$

C $-0.0115987244-0.0112440373-2.6403759059$

C $0.0733775042-0.0271050073-4.1077118125$

H $0.20382384340 .982783476-4.5179540792$

H $-0.8341546978-0.4462560174-4.561317838$

H $0.9198986798-0.631156107-4.4594540498$

11ts MeCC- + HCCMe, ts, DFT

H -0.71905638630 .72567586384 .5115294556$

$\begin{array}{llll}\text { C } & -0.0075852779 & -0.0031749508 & 4.099292579\end{array}$

H 0.97865182920 .24746868524 .51388938

C $-0.0051148057-0.001899727 \quad 2.6354281801$

H $-0.2843221291 \quad-0.98373361264 .5107364343$

C $-0.0028518458-0.0007115701 \quad 1.4089224857$

H -0.00011753130 .00035588520 .0000000355$

C $0.00266035450 .001299207-1.4089223756$

C $0.0049336760 .0020253006-2.6354283524$

$\begin{array}{llll}\text { C } & 0.0077935713 & 0.0027173354 & -4.0992913752\end{array}$

H $0.76019749270 .6899880507 \quad-4.5102722642$

H $-0.962240756 \quad 0.3102486227-4.513810989$

H $0.2278734462-0.991537066-4.5120789015$

$\underline{\text { Non-identity Proton Transfers }}$

16d See entry for $\mathbf{8 d}$.

16a See entry for 9 a.

16ts acetonitrile anion + acetaldehyde, ts, HF

H $0.8222744730 .8536377527-1.517501222$

$\begin{array}{llll}\text { C } & 0.8646015702 & 1.0490003168 & -0.4507251686\end{array}$

H $1.8736048073 \quad 1.0452875092 \quad-0.0518926341$

H $0.2695715735-0.09118605810 .1075368339$

$\begin{array}{llll}\text { C } & 0.0723749882 & 2.1752915326 & -0.0440025686\end{array}$

$\begin{array}{lllll}\text { O } & 0.2203517455 & 2.884757322 & 0.9251206733\end{array}$

H $-0.82115290252 .3475369586-0.6721512598$ 


$$
\begin{array}{llll}
\text { C } & -0.2723639338 & -1.32836668 & 0.7243151583 \\
\text { H } & 0.4424465489 & -1.6586451413 & 1.4717981495 \\
\text { C } & -0.4749475058 & -2.3491485341 & -0.2651418327 \\
\text { H } & -1.2135944458 & -1.0627619906 & 1.1963863477 \\
\text { N } & -0.6105649617 & -3.113226774 & -1.1031164474
\end{array}
$$

16ts acetonitrile anion + acetaldehyde, ts, MP2

$\begin{array}{lrrr}\mathrm{H} & 0.608444 & -1.697635 & 0.445780 \\ \mathrm{C} & 1.029927 & -0.701482 & 0.600316 \\ \mathrm{H} & 1.290916 & -0.489471 & 1.639758 \\ \mathrm{H} & 0.015169 & 0.091704 & 0.359546 \\ \mathrm{C} & 2.063667 & -0.360266 & -0.346526 \\ \mathrm{O} & 2.982912 & 0.458111 & -0.195961 \\ \mathrm{H} & 1.936456 & -0.828572 & -1.353025 \\ \mathrm{C} & -1.238363 & 1.006215 & 0.174249 \\ \mathrm{H} & -1.367790 & 1.586222 & 1.091979 \\ \mathrm{C} & -2.351000 & 0.155779 & -0.083838 \\ \mathrm{H} & -1.007946 & 1.654592 & -0.675674 \\ \mathrm{~N} & -3.194847 & -0.654744 & -0.286556\end{array}$

16ts acetonitrile anion + acetaldehyde, ts, DFT

H $\quad 0.667330 \quad-1.748388 \quad-0.050384$

$\begin{array}{llll}\text { C } & 1.050824 & -0.807746 & 0.350439\end{array}$

$\mathrm{H} \quad 1.158996-0.809824 \quad 1.436982$

$\mathrm{H} \quad-0.005533 \quad 0.003724 \quad 0.169092$

C $\quad \begin{array}{llll}2.181181 & -0.282110 & -0.362423\end{array}$

$\begin{array}{llll}\mathrm{O} & 3.051078 & 0.490890 & 0.057971\end{array}$

$\mathrm{H} \quad 2.203880 \quad-0.553941 \quad-1.448184$

$\begin{array}{llll}\text { C } & -1.239744 & 0.913169 & 0.076023\end{array}$

$\mathrm{H} \quad-1.200181 \quad 1.516251 \quad 0.984990$

$\begin{array}{llll}\text { C } & -2.433133 & 0.161980 & -0.038383\end{array}$

$\mathrm{H} \quad-1.029779 \quad 1.517738 \quad-0.808740$

$\mathrm{N} \quad-3.365443 \quad-0.537777 \quad-0.128779$

17d See entry for 9d.

17a See entry for $\mathbf{8 a}$.

17ts See entry for $\mathbf{1 6 t s .}$

18d See entry for 10d.

18a See entry for $9 \mathbf{a}$.

18ts acetonitrile anion + nitromethane, ts, HF

H $-0.05944014880 .7993969333-1.6807527427$ 
C $0.4553820140 .7301103107-0.735380604$

H $1.53004370350 .6809760716-0.814522101$

H $0.0113712503-0.3856148312-0.0732682806$

H - $-1.54293196-1.5300181280 .755367362$

N $0.1000258106 \quad 1.85139498260 .0699210333$

N $0.169296241-3.6449781756-0.8094587191$

C $-0.4670177179-1.60922157290 .6364749764$

H $0.0180609147-1.6467625951 .6066757496$

C $-0.1300501512-2.769436544 \quad-0.1404999544$

$\begin{array}{llllllll}\text { O } & 0.8618023791 & 2.2141867974 & 0.929735098\end{array}$

O $-0.98583275292 .3518621698-0.0772729348$

18ts acetonitrile anion + nitromethane, ts, MP2

H $-0.0729998920 .7305142807 \quad-1.686816513$

$\begin{array}{lllll}\text { C } & 0.4505832928 & 0.6873539421 & -0.7339633358\end{array}$

$\begin{array}{lllll}\text { H } & 1.5330898371 & 0.6100811486 & -0.8123195696\end{array}$

H $0.022256955-0.3396922329-0.0888170139$

H $-1.573071261 \quad-1.49553920310 .7855099569$

N 0.1025298891 .81915211590 .061091918

N $0.2221372446-3.554766199-0.8915917282$

$\begin{array}{llll}\text { C } & -0.4908355913 & -1.5996099618 & 0.6792604068\end{array}$

H $0.0096797049-1.61308366841 .6501633437$

C $-0.1292259075-2.7115596507-0.1337794491$

$\begin{array}{lllll}\text { O } & 0.8865511265 & 2.1780597007 & 0.9662222589\end{array}$

O $-1.03339563192 .3219293343-0.0791381668$

18ts acetonitrile anion + nitromethane, ts, DFT

H $-0.08454029230 .7148433367-1.6506798619$

$\begin{array}{llll}\text { C } & 0.4399506791 & 0.7071479045 & -0.6995618867\end{array}$

H $1.51841322960 .5985162956-0.7695233646$

H $0.0132703769-0.3580249948-0.063510549$

H $-1.5426332389-1.5002538745 \quad 0.7515099252$

$\begin{array}{llll}\mathrm{N} & 0.1026803434 & 1.853295841 & 0.0652170475\end{array}$

N $0.1876363334-3.6396166622-0.8571867266$

C $-0.4671909928-1.64425460750 .642710992$

H $0.0424583271-1.6227719971 \quad 1.6065335383$

C $-0.1290624022-2.7690354892-0.1453230751$

$\begin{array}{lllll}\text { O } & 0.8911007909 & 2.2367027437 & 0.9568594198\end{array}$

O $-1.02127189642 .3768960233-0.0965466842$

19d See entry for 9d.

19a See entry for 10a.

19ts See entry for 18ts. 


\section{Identity Hydride Transfers to Carbonyls}

20d formate, $\mathrm{HF}$

H 0. 0. -1.4311725845

C $0.0 . \quad-0.3089942787$

O $1.1169977267 \quad 0.0 .2053211411$

O -1.1169977267 0. 0.2053211411

20d formate, MP2

H 0. 0. -1.4514374017

C 0. 0. -0.3176294125

O $1.1419592594 \quad 0.0 .2098258673$

O -1.1419592594 0. 0.2098258673

20d formate, DFT

H 0. 0. -1.4529238145

C 0. $0 .-0.3149445047$

$\begin{array}{llllll}\text { O } & 1.1373676584 & 0 . & 0.2089119277\end{array}$

O -1.1373676584 0. 0.2089119277

20a co2, carbon dioxide, $\mathrm{HF}$

O $0.0 .-1.1357329018$

C 0. 0.0 .

O 0.0 .1 .1357329018

20a co2, carbon dioxide, MP2

$\begin{array}{llll}\mathrm{O} & 0.000000 & 0.000000 & -1.170031\end{array}$

$\begin{array}{llll}\mathrm{C} & 0.000000 & 0.000000 & 0.000000\end{array}$

$\begin{array}{llll}\mathrm{O} & 0.000000 & 0.000000 & 1.170031\end{array}$

20a co2, carbon dioxide, DFT

O $0.0 . \quad-1.160799672$

C 0.0 .0 . 0 .

O

20ts $\mathrm{CO} 2+$ formate ts, $\mathrm{HF}$

O $1.7316439737-0.0137489653-1.1279750358$

C $1.3849967258-0.01091714210$.

H 0.0 .0 .

$\begin{array}{lllll}\text { O } & 1.7316455406 & -0.0135501776 & 1.1279750358\end{array}$

C -1.38499672580 .01091714210$.

$\begin{array}{lllll}\text { O } & -1.7316455406 & 0.0135501776 & 1.1279750358\end{array}$

$\begin{array}{llll}\text { O } & -1.7316439737 & 0.0137489653 & -1.1279750358\end{array}$ 
20ts $\mathrm{CO} 2+$ formate ts, MP2

O $1.725079-0.013276-1.1573924222$

C $1.348369-0.0106280$.

H 0.0 .0 .0000065778

O $1.725083-0.01392 \quad 1.1573915778$

C -1.3483690 .0106280$.

O $-1.725083 \quad 0.01392 \quad 1.1573915778$

O $-1.7250790 .013276-1.1573924222$

20ts $\mathrm{CO} 2+$ formate ts, DFT

O $1.7431598876-0.0138341282-1.1494513785$

C $1.3665437872-0.01077168810$.

H 0. 0. -0.0000000754

O $1.7431613024 \quad-0.0136465603 \quad 1.1494513851$

C -1.36654378720 .01077168810$.

$\begin{array}{lllll}\text { O } & -1.7431613024 & 0.0136465603 & 1.1494513851\end{array}$

O $-1.7431598876 \quad 0.0138341282-1.1494513785$

21d thioformate-, HF

O $0.2934307507 \quad 0 .-1.5871936577$

C -0.3895090209 0. -0.5961397862

S 0.09211479550 .1 .0630812207

H -1.4842286077 0. -0.7349115522

21d thioformate-, MP2

O 0.3113565418 0. -1.6021917399

C -0.3932851343 0. -0.5786540964

S $0.0856433137 \quad 0.1 .0631997982$

H -1.5014345482 0. -0.721738274

21d thioformate-, DFT

O $0.30615194010 .-1.6063425356$

C $-0.392578386 \quad 0 .-0.5915575373$

S 0.0879811719 0. 1.0705925807

H $-1.5014439551 \quad 0 .-0.7293957819$

21a carbon oxysulfide, $\mathrm{HF}$

O 0. 0. -1.6601376966

C 0. $0 .-0.5358242039$

S 0. 0.1 .0310029248

21a carbon oxysulfide, MP2 (complex)

O $0.0 . \quad-1.6918461527$

C 0. $0 .-0.5213321713$ 
S 0. 0.1 .0414226406

21a carbon oxysulfide, DFT

O 0 . $0 .-1.6848707728$

C 0. 0. -0.5277042723

S 0.0 .1 .0403244885

21ts (anti) COS + HCOS-, ts, anti, HF

O $-1.7195171763-0.0058238189-1.0968662964$

C $-0.5683707257-0.001644014-1.2256824104$

H $0.0000281855-0.0000954081-0.0000265201$

$\begin{array}{lllll}\text { S } & 0.6541231728 & 0.0032640667 & -2.3518614858\end{array}$

C $0.5683437095 \quad 0.00133097891 .2256573996$

S $-0.6540964217 \quad-0.0027424512 \quad 2.3519014881$

O $1.719480413 \quad 0.0050272902 \quad 1.0968083648$

21ts (anti) COS + HCOS-, anti, MP2 (a complex)

O $-1.75196309-0.0058308388-1.0854866103$

C $-0.5491927122-0.0016293856-1.2040830828$

H $0.0000874238-0.0005548964-0.0000171662$

S $0.6630721546 \quad 0.0032477609-2.3174234508$

C $0.5492379065 \quad 0.00126881941 .2041056077$

S $-0.6631216932-0.00278909872 .317353514$

O $1.75201734340 .0052533011 \quad 1.085611736$
21ts (anti) COS + HCOS-, ts, anti, DFT
O $-1.7467300026-0.0054859876-1.1077007371$
C $-0.5573262202-0.0014545644-1.2264171713$
H 0.00000119140 .00001812110 .0000025463
S $\quad 0.65540850420 .0029855276 \quad-2.3613930579$
$\begin{array}{llll}\text { C } & 0.557324822 & 0.0014781152 & 1.2264133485\end{array}$
S $-0.655408099-0.00302750152 .361393335$
O $1.74673009180 .0055500073 \quad 1.1077027316$

22ts (syn) COS + HCOS-, ts, syn, HF

O $-1.6061743173-1.11456852050 .9114264369$

C $-0.7911223407-0.3144235395 \quad 1.0994527075$

H $-0.0857270352-0.0893514292-0.0338473599$

S $-0.14118185740 .6874353663 \quad 2.2581186451$

C $0.1799325778-0.3217722533-1.3407503414$

S $1.45041742290 .6752818887-1.741243053$

O $-0.543188612-1.1225502162-1.7599734757$

22ts (syn) COS + HCOS-, syn, MP2 (a complex)

O $-1.6704829087-1.14610064610 .9841261166$ 
$\begin{array}{llll}\text { C } & -0.7945544445 & -0.3205029747 & 1.0907088813 \\ \text { H } & -0.1660425012 & -0.1721770203 & -0.0655569002 \\ \text { S } & -0.0778572027 & 0.7078534891 & 2.157295436 \\ \text { C } & 0.1714234891 & -0.3278352949 & -1.3367179226 \\ \text { S } & 1.4271292576 & 0.6964237614 & -1.6246049723 \\ \text { O } & -0.5399576719 & -1.1546780253 & -1.8568056505 \\ \text { 22ts }(\text { syn) } & \text { COS + HCOS-, ts, syn, DFT } \\ \text { O } & -1.6732917973 & -1.1418337393 & 1.002874573 \\ \text { C } & -0.8075313881 & -0.3250886333 & 1.1106348123 \\ \text { H } & -0.1671290671 & -0.1738992274 & -0.0659456468 \\ \text { S } & -0.0949475267 & 0.7076741909 & 2.1992626997 \\ \text { C } & 0.1756909221 & -0.3325640754 & -1.3601550804 \\ \text { S } & 1.443535852 & 0.6959952095 & -1.6668192206 \\ \text { O } & -0.5291133704 & -1.1505281267 & -1.8723781243\end{array}$

23d methoxide-, $\mathrm{HF}$

C 0. 0. -0.5461449378

O 0.0 .0 .7978943521

H $0.1 .0220939949-1.0354283968$

H $-0.8851593646-0.5110469974-1.0354283968$

H $0.8851593646-0.5110469975-1.0354283968$

23d methoxide-, MP2

C 0. 0. -0.5391883295

O 0.0 .0 .7936494867

H $0.1 .0272841107-1.0380219722$

H $-0.8896541368-0.5136420553-1.0380219722$

H $0.8896541368-0.5136420554-1.0380219722$

23d methoxide-, DFT

C 0. 0. -0.5390128235

O 0.0 .0 .7935751765

H $0.1 .0271009412-1.0381748235$

H $-0.8894955073-0.5135504706-1.0381748235$

H $0.8894955073-0.5135504706-1.0381748235$

23a formaldehyde, $\mathrm{HF}$

H $0.9375410 .-1.118130875$

C 0. $0 . \quad-0.533425875$

O 0.0 .0 .679602125

H $-0.9375410 .-1.118130875$

23a formaldehyde, MP2

$\begin{array}{llll}\mathrm{H} & 0.937541 & 0.000000 & -1.107937\end{array}$

$\begin{array}{llll}\text { C } & 0.000000 & 0.000000 & -0.523232\end{array}$

$\begin{array}{llll}\mathrm{O} & 0.000000 & 0.000000 & 0.689796\end{array}$ 


\begin{tabular}{lccc}
$\mathrm{H}$ & -0.937541 & 0.000000 & -1.107937 \\
23a & \multicolumn{3}{c}{ formaldehyde, DFT } \\
$\mathrm{H}$ & 0.940086 & 0.000000 & -1.116160 \\
$\mathrm{C}$ & 0.000000 & 0.000000 & -0.529804 \\
$\mathrm{O}$ & 0.000000 & 0.000000 & 0.672040 \\
$\mathrm{H}$ & -0.940086 & 0.000000 & -1.116160
\end{tabular}

23ts methoxide + formaldehyde, ts, HF

$\begin{array}{llll}\text { O } & -2.1945276061 & 0.4148435152 & -0.0090008485\end{array}$

C $-1.1391476061-0.2641924848 \quad-0.0119768485$

H $-0.8602536061-0.84702648480 .8821161515$

H -0.02975960610 .46255051520 .0070271515$

H $-0.8475236061 \quad-0.8193334848-0.9195268485$

C 1.38601939390 .45936851520 .0168401515

O $1.8693093939 \quad-0.6799624848 \quad 0.0024631515$

H 1.42268239391 .06268251520 .9370781515

H $1.43536939391 .0910225152-0.8835728485$

23ts methoxide + formaldehyde ts, MP2

O $-2.0920260606 \quad 0.6034055152-0.0122341515$

C $-1.2634640606-0.3644794848-0.0210251515$

H $-1.1577030606-1.01047248480 .8868698485$

H $0.00001093940 .0001275152-0.0000031515$

H $-1.1370330606-0.9760654848 \quad-0.9498641515$

C $1.2635289394 \quad 0.3645395152 \quad 0.0210398485$

$\begin{array}{lllll}\text { O } & 2.0919289394 & -0.6034884848 & 0.0122198485\end{array}$

H $1.1372289394 \quad 0.97615651520 .9498758485$

H $1.15788393941 .0105575152-0.8868521515$

23ts methoxide + formaldehyde ts, DFT

O $-2.1143827482 \quad 0.5920670362-0.0148032812$

C $-1.290272416-0.3744140244-0.0236546261$

H $-1.1898781744 \quad-1.02250210320 .8827335833$

H $-0.000246632-0.00005814620 .0001979162$

H $-1.166524453-0.9858077384-0.9523706479$

C $1.2903643558 \quad 0.3744023135 \quad 0.024264288$

O $2.1143984613 \quad-0.5920054756 \quad 0.0139650332$

H $1.1666087665 \quad 0.98448766340 .9538120978$

H $1.1893631491 .0234581047 \quad-0.8813249367$

24d formamide, $\mathrm{H}$ - adduct, $\mathrm{HF}$

O $-0.19412608-0.58316588-1.08157524$ 
$\begin{array}{lrrr}\text { C } & 0.42591492 & -0.30807588 & 0.05951976 \\ \text { H } & 1.48263592 & 0.06136012 & -0.04890424 \\ \text { H } & 0.48108392 & -1.16134788 & 0.79012976 \\ \text { N } & -0.21330608 & 0.79305212 & 0.90102176 \\ \text { H } & -0.29020108 & 1.57610512 & 0.24959776 \\ \text { H } & -1.18285708 & 0.48630012 & 0.99750776\end{array}$

24d formamide, $\mathrm{H}$ - adduct, MP2

O $\quad-0.194914 \quad-0.581824 \quad-1.080686$

$\begin{array}{llll}\text { C } & 0.425127 & -0.306734 & 0.060409\end{array}$

$\begin{array}{llll}\mathrm{H} & 1.481848 & 0.062702 & -0.048015\end{array}$

H $\quad 0.480296 \quad-1.160006 \quad 0.791019$

$\begin{array}{llll}\mathrm{N} & -0.214094 & 0.794394 & 0.901911\end{array}$

$\begin{array}{llll}\mathrm{H} & -0.290989 & 1.577447 & 0.250487\end{array}$

$\mathrm{H} \quad-1.183645 \quad 0.487642 \quad 0.998397$

24d formamide, $\mathrm{H}$ - adduct, DFT

O $-0.1859035022 \quad-0.5907937013-1.082716375$

C $0.4241838508 \quad-0.3096491628 \quad 0.055012086$

H $1.48581403 \quad 0.0604353631-0.0474376455$

H $0.4839856784-1.16275172640 .7920015756$

$\mathrm{N}-0.2201419143 \quad 0.79928940590 .9020314865$

H $-0.2956987934 \quad 1.59210971180 .2616411404$

H -1.1909826019 $0.4994253976 \quad 1.0112330083$

24a formamide, $\mathrm{HF}$

N $0.1682217083 \quad 0 .-1.0855661667$

C -0.3908362917 0. 0.1588528333

O 0.2366767083 0. 1.2015098333

H $-1.4947682917 \quad 0.0 .1223808333$

H 1.1725677083 0. -1.1756701667

H -0.4037472917 0. -1.9129431667

24a formamide, MP2

$\begin{array}{llll}\mathrm{N} & 0.171361 & 0.000000 & -1.077495\end{array}$

$\begin{array}{llll}\text { C } & -0.387697 & 0.000000 & 0.166924\end{array}$

$\begin{array}{llll}\mathrm{O} & 0.239816 & 0.000000 & 1.209581\end{array}$

$\begin{array}{llll}\mathrm{H} & -1.491629 & 0.000000 & 0.130452\end{array}$

$\mathrm{H} \quad \begin{array}{llll}1.175707 & 0.000000 & -1.167599\end{array}$

$\mathrm{H} \quad-0.400608 \quad 0.000000 \quad-1.904872$

24a formamide, DFT

N $0.16816932620 . \quad-1.0828568933$

C -0.3882528937 0. 0.1590093213

O 0.2343281636 0. 1.1985786951

H - 1.49364419120 .0 .1256704203 


\begin{tabular}{lrrr} 
H & 1.173102434 & 0. & -1.1756906223 \\
$\mathrm{H}$ & -0.4017514724 & 0. & -1.9126670332 \\
\multicolumn{4}{c}{ formamide H- ts, HF } \\
\begin{tabular}{lrrr} 
24ts & $(\mathrm{R}, \mathrm{R})$ & \multicolumn{3}{c}{ formam } \\
$\mathrm{H}$ & -1.359767 & -0.488568 & -1.423316 \\
$\mathrm{~N}$ & -0.464642 & -0.592301 & -1.866039 \\
$\mathrm{H}$ & -0.206275 & -1.557849 & -1.816587 \\
$\mathrm{C}$ & 0.533324 & 0.163555 & -1.178915 \\
$\mathrm{H}$ & 0.391945 & 1.237743 & -1.361972 \\
$\mathrm{O}$ & 1.691187 & -0.297205 & -1.098233 \\
$\mathrm{H}$ & -0.016782 & 0.242746 & 0.080117 \\
$\mathrm{C}$ & -0.599517 & -0.338617 & 1.183233 \\
$\mathrm{H}$ & -0.366818 & 0.524493 & 1.822166 \\
$\mathrm{~N}$ & 0.311590 & -1.407536 & 1.442015 \\
$\mathrm{O}$ & -1.791793 & -0.609223 & 0.929249 \\
$\mathrm{H}$ & -0.032117 & -2.223370 & 0.975369 \\
$\mathrm{H}$ & 1.221522 & -1.199637 & 1.072075
\end{tabular}
\end{tabular}

24ts (R,R) formamide H- ts, MP2

$\mathrm{H}-1.3043033878-0.0247546735-1.1918949184$

$\mathrm{N}-0.4299763878-0.0705206735-1.7293749184$

H $-0.1235083878-1.0413876735-1.6988409184$

C $0.58961961220 .6878703265-1.0274629184$

H $0.45935961221 .7750743265-1.2363469184$

$\begin{array}{lllll}\text { O } & 1.7658486122 & 0.2055763265 & -0.9377299184\end{array}$

H $0.0355856122 \quad 0.74657732650 .1756350816$

C $-0.5469393878 \quad 0.2089523265 \quad 1.2384200816$

H $-0.3238533878 \quad 1.07310232651 .9063200816$

N $0.3851416122-0.87083867351 .5080230816$

O $-1.7596113878-0.07502667350 .9683400816$

H $-0.0066923878-1.69478067351 .0552270816$

H $1.2712766122-0.66964867351 .0287390816$

24ts (R,R) formamide H- ts, DFT

$\mathrm{H}-1.3189428341-0.0003951374-1.2761464706$

$\mathrm{N}-0.4323993404-0.0424331501-1.7868215215$

H $-0.1389487248-1.0167761588-1.7935453003$

C $0.58946084360 .6924786775-1.0603687357$

H $0.46500535941 .7854954348-1.2395566973$

O $1.7623351525 \quad 0.2064418954-0.986420855$

H 0.03446007010 .7241822710 .1703454742

C $-0.54706760490 .1984182093 \quad 1.2699287112$

H $-0.3285815141 \quad 1.08147993361 .9139388052$

N $0.3889072529-0.8715507957 \quad 1.5718317494$

O $-1.7570911675 \quad-0.0962441127 \quad 1.0123406819$

H 0.0088933095 - 1.71635615671 .1506476362 
H $1.286247634-0.68670614821 .1145264895$

\begin{tabular}{llll} 
25ts $(\mathrm{R}, \mathrm{S})$ & \multicolumn{4}{c}{ formamide H- transfer, ts, HF } \\
H & 1.4709312653 & 0.9233426735 & -1.2838318367 \\
N & 1.7601222653 & 1.1458426735 & -0.3302508367 \\
H & 2.4519112653 & 0.4583526735 & -0.0390148367 \\
C & 0.6135892653 & 1.0071846735 & 0.5608961633 \\
O & 0.8162002653 & 0.7842896735 & 1.7914051633 \\
H & -0.0017017347 & -0.0056673265 & 0.0051561633 \\
H & -0.1729047347 & 1.7403386735 & 0.2430821633 \\
C & -0.6131597347 & -1.0108143265 & -0.5466208367 \\
H & 0.1503592653 & -1.7549423265 & -0.1989178367 \\
O & -0.7810717347 & -0.8018553265 & -1.7864678367 \\
N & -1.7887867347 & -1.1263913265 & 0.3121751633 \\
H & -1.5231077347 & -0.9095613265 & 1.2737511633 \\
H & -2.4584417347 & -0.4257193265 & 0.0011541633
\end{tabular}

25ts (R,S) formamide H- transfer, ts, MP2

$\mathrm{H} \quad 1.455293 \quad 0.937919 \quad-1.281909$

$\begin{array}{llll}\mathrm{N} & 1.744484 & 1.160419 & -0.328328\end{array}$

$\begin{array}{llll}\mathrm{H} & 2.436273 & 0.472929 & -0.037092\end{array}$

$\begin{array}{llll}\text { C } & 0.597951 & 1.021761 & 0.562819\end{array}$

$\begin{array}{llll}\mathrm{O} & 0.800562 & 0.798866 & 1.793328\end{array}$

$\begin{array}{llll}\mathrm{H} & -0.017340 & 0.008909 & 0.007079\end{array}$

$\begin{array}{llll}\mathrm{H} & -0.188543 & 1.754915 & 0.245005\end{array}$

$\begin{array}{llll}\text { C } & -0.628798 & -0.996238 & -0.544698\end{array}$

$\mathrm{H} \quad 0.134721 \quad-1.740366-0.196995$

$\begin{array}{llll}\mathrm{O} & -0.796710 & -0.787279 & -1.784545\end{array}$

$\begin{array}{llll}\mathrm{N} & -1.804425 & -1.111815 & 0.314098\end{array}$

$\mathrm{H} \quad \begin{array}{llll}-1.538746 & -0.894985 & 1.275674\end{array}$

$\begin{array}{llll}\mathrm{H} & -2.474080 & -0.411143 & 0.003077\end{array}$

25ts (R,S) formamide H- transfer, ts, DFT

H $1.03927791290 .9264668234-1.3294026217$

N $1.4569241666 \quad 1.3031082104-0.4763285661$

H $2.3575359120 .8482384645 \quad-0.341572697$

C 0.62578150520 .93428603860 .6818406669

$\begin{array}{lllll}\text { O } & 1.2068204665 & 0.7899389828 & 1.8124704817\end{array}$

H $0.0837535826 \quad-0.1380673765 \quad 0.2926077625$

H $-0.3074497342 \quad 1.5638596213 \quad 0.6401017174$

C $-0.6873679615-1.1500206833-0.4536810259$

H $-0.1506339471-1.96382219930 .0805431454$

O $-0.5105758753-0.9504891993-1.678090924$

$\begin{array}{llll}\mathrm{N} & -2.0007362766 & -0.9701210167 & 0.1115272809\end{array}$

H $-1.9586634517-0.8026879895 \quad 1.1132188093$

H $-2.4575734959-0.1860880995-0.3458814267$ 
Other Identity Hydride Transfers

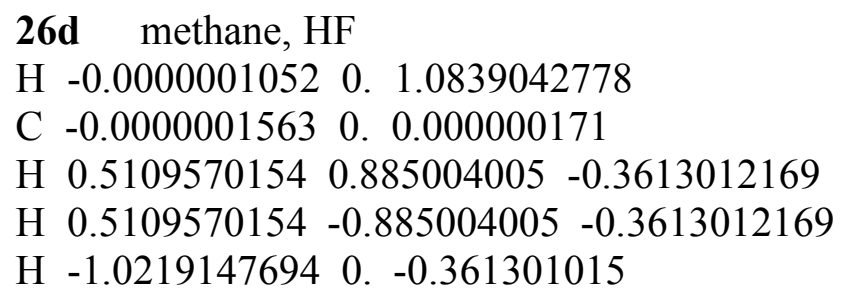

26d methane, $\mathrm{HF}$

$\mathrm{H}-0.0000001052 \quad 0.1 .0839042778$

C -0.0000001563 0. 0.000000171

H $0.51095701540 .885004005-0.3613012169$

H $0.5109570154-0.885004005-0.3613012169$

H $-1.0219147694 \quad 0 .-0.361301015$

26d methane, MP2

$\begin{array}{llll}\mathrm{H} & 0.000000 & 0.000000 & 1.090444\end{array}$

$\begin{array}{llll}\text { C } & 0.000000 & 0.000000 & 0.000000\end{array}$

$\mathrm{H} \quad 0.514040 \quad 0.890344 \quad-0.363481$

$\mathrm{H} \quad \begin{array}{llll}0.514040 & -0.890344 & -0.363481\end{array}$

$\mathrm{H} \quad-1.028081 \quad 0.000000 \quad-0.363481$

26d methane, DFT

H 0. 0. 1.090444

C 0. 0. 0.

H $0.514040 .890344 \quad-0.363481$

H $0.51404-0.890344-0.363481$

H $-1.0280810 .-0.363481$

26a methyl+, HF

H $-0.00000018550 .-1.0817415231$

C 0. 0. 0 .

H 0.93681573210 .0 .5408706009

H $-0.9368155466 \quad 0.0 .5408709222$

26a methyl+, MP2

H $-0.00000018720 .-1.091428$

C 0. 0.0.

H 0.9452044680 .0 .5457138379

H $\quad-0.9452042808 \quad 0.0 .5457141621$

26a methyl+, DFT,

H -0.0000001876 0. -1.093601868

C 0. 0. 0 .

H 0.94708709310 .0 .5468007715

H $-0.9470869055 \quad 0.0 .5468010964$

26cx methane + methyl $^{+}$, complex, $\mathrm{HF}$

$\mathrm{H} \quad-0.260519 \quad-1.094268 \quad 0.991430$

$\begin{array}{llll}\text { C } & -0.014398 & -0.050206 & 1.044157\end{array}$

$\begin{array}{llll}\mathrm{H} & -0.751355 & 0.538731 & 1.575785\end{array}$ 


$\begin{array}{cccc}\mathrm{H} & -0.237731 & 0.595832 & 0.008899 \\ \mathrm{H} & 1.007010 & 0.177895 & 1.294332 \\ \mathrm{C} & 0.033572 & 0.007719 & -1.048808 \\ \mathrm{H} & -0.865515 & -0.525445 & -1.304323 \\ \mathrm{H} & 0.933582 & -0.577866 & -1.026761 \\ \mathrm{H} & 0.152243 & 0.959070 & -1.552281\end{array}$

\begin{tabular}{lrrr} 
26cx & \multicolumn{4}{c}{ methane + methyl $^{+}$, complex, MP2 } \\
H & -0.309204 & -1.092221 & 0.962021 \\
C & -0.009467 & -0.050396 & 0.964558 \\
H & -0.736444 & 0.565492 & 1.509372 \\
H & -0.274900 & 0.685072 & 0.012664 \\
H & 1.019992 & 0.146524 & 1.246119 \\
C & 0.029337 & 0.000937 & -0.965573 \\
H & -0.854331 & -0.559384 & -1.253700 \\
H & 0.965975 & -0.544252 & -0.992539 \\
H & 0.121991 & 0.965414 & -1.481154
\end{tabular}

26cx methane + methyl $^{+}$, complex, DFT

H $-0.2793630709-1.09253141210 .9928393381$

C $-0.014847212-0.04251049651 .0134932835$

$\mathrm{H}-0.7609554441 \quad 0.558824058 \quad 1.5440974945$

H -0.26079596640 .64927041380 .0120444993$

H $1.0121683823 \quad 0.18089273391 .2814918446$

C $0.02710099940 .0119595699-1.0140602651$

H $-0.8727224185-0.5280726709-1.2882417666$

H $0.9442939703 \quad-0.5638979199-1.0234012327$

H $0.14385182260 .978820357-1.5154282879$

27d 1,4-dihydropyridine, DFT

C $-1.1925877475 \quad 0.11532573530 .69677585$

H $-0.0045918279-0.01814133842 .3833990012$

C $1.1931470642 \quad-0.0856223586 \quad 0.6999836064$

N $0.0135627336 \quad 0.184431924 \quad 1.3978863883$

C $-1.24389181590 .0211652306-0.6367691296$

H -2.08511423820 .15694958991 .309157968$

H $-0.0669722599-0.830415063-2.2319192703$

H $-2.2140589976-0.0141787965-1.1180858733$

C $1.231641726-0.1874745834-0.6334044693$

H $2.0783174961 \quad-0.1933392544 \quad 1.314972746$

H $2.1836017651-0.3847185796-1.1119864778$

C $0.00083169-0.015060753-1.4978484141$

H $0.0790334273 \quad 0.9028203492 \quad-2.1031674725$

27a pyridinium, DFT

H 0. $-0.0000006396-2.4963882626$ 
C 0. $-0.0000003441-1.4127812203$

$\mathrm{N}$ 0. 0.00000035771 .3074866351

C 0. $-1.2096747159-0.715525231$

C $0.1 .2096743234-0.7155258202$

C $0.1 .1883330108 \quad 0.6652658456$

C $0 .-1.18833267820 .6652664476$

H $0 .-2.1600146764-1.232486483$

H 0. $2.1600140192-1.2324875365$

H 0. 2.0766510641 .2825507368

H $0 .-2.07665042331 .2825518846$

H 0.0 .00000058322 .3236530852

27ts dihydropyridine + pyridinium, ts, DFT

$\begin{array}{llll}\mathrm{H} & 1.533361 & 0.706304 & 1.423733\end{array}$

$\begin{array}{llll}\text { C } & 0.747395 & 1.130967 & 0.797021\end{array}$

$\begin{array}{llll}\text { C } & -0.046349 & 2.199134 & 1.382119\end{array}$

$\begin{array}{llll}\mathrm{H} & 0.000000 & 0.000000 & 0.855598\end{array}$

$\begin{array}{llll}\text { C } & 1.058746 & 1.296616 & -0.612400\end{array}$

$\begin{array}{llll}\text { C } & 0.435734 & 2.253891 & -1.348296\end{array}$

$\mathrm{H} \quad 1.786760 \quad 0.657331 \quad-1.092986$

$\begin{array}{llll}\mathrm{N} & -0.435734 & 3.132591 & -0.763951\end{array}$

$\begin{array}{llll}\mathrm{H} & 0.620186 & 2.397966 & -2.404542\end{array}$

$\begin{array}{llll}\text { C } & -0.638669 & 3.130576 & 0.594800\end{array}$

$\mathrm{H} \quad-0.187200 \quad 2.251080 \quad 2.453713$

$\mathrm{H} \quad-1.259245 \quad 3.926265 \quad 0.984106$

$\mathrm{H} \quad-0.851623 \quad 3.862616 \quad-1.323628$

$\begin{array}{llll}\text { C } & -0.747395 & -1.130967 & 0.797021\end{array}$

$\begin{array}{llll}\text { C } & 0.046349 & -2.199134 & 1.382119\end{array}$

$\begin{array}{llll}\text { C } & -1.058746 & -1.296616 & -0.612400\end{array}$

$\mathrm{H} \quad-1.533361 \quad-0.706304 \quad 1.423733$

C $\quad-0.435734 \quad-2.253891 \quad-1.348296$

$\mathrm{H} \quad-1.786760 \quad-0.657331 \quad-1.092986$

$\mathrm{N} \quad \begin{array}{llll}0.435734 & -3.132591 & -0.763951\end{array}$

$\mathrm{H} \quad-0.620186-2.397966 \quad-2.404542$

$\begin{array}{llll}\text { C } & 0.638669 & -3.130576 & 0.594800\end{array}$

$\mathrm{H} \quad 0.187200 \quad-2.251080 \quad 2.453713$

$\mathrm{H} \quad 1.259245 \quad-3.926265 \quad 0.984106$

$\mathrm{H} \quad 0.851623 \quad-3.862616 \quad-1.323628$

28d $\operatorname{chf} 3, \mathrm{HF}$

H 0. 0. -1.4096014355

C 0. 0. -0.3332316966

$\begin{array}{llll}\text { F } & 0.615157312 & 1.0654837191 & 0.1262589487\end{array}$

F $0.615157312-1.06548371910 .1262589487$

F $-1.2303146241 \quad 0.0 .1262589487$ 

28d $\operatorname{chf} 3, \mathrm{MP} 2$
H 0. 0. -1.4266721731
C 0. 0. -0.3389159313
F $0.6267689035 \quad 1.0855955855 \quad 0.1281543615$
F $0.6267689035-1.08559558550 .1281543615$
F $-1.253537807 \quad 0.0 .1281543615$

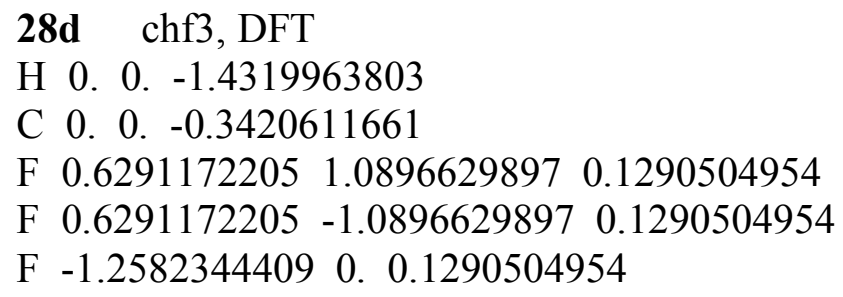

28ts chf3h, hydride ts, HF

H $-0.0005043592-0.0002595094-0.0000097034$

C $-0.0235042077 \quad 0.0117479573 \quad 1.3150092779$

F $-0.7104757654 \quad 1.0319183602 \quad 1.5568996096$

F $1.19446134790 .0964103112 \quad 1.5988388457$

F $-0.5683573797 \quad-1.0858875409 \quad 1.5786941699$

C $0.023394039-0.0118022191-1.3150059519$

F $-0.5117283782-1.1143230788-1.5782494117$

F $1.2507094497 \quad 0.0685057924-1.5566973943$

F -0.65447978861 .0034411648 - 1.5994869583

28ts chf3h, hydride ts, MP2

H 0.00090029220 .00032275370 .0000124156

C -0.02327816550 .01170770791 .3066056126$ 
F $-0.7269176518 \quad 1.0516494454 \quad 1.5586434022$

F $1.2194836254 \quad 0.1005492904 \quad 1.6028421542$

F $-0.577568989-1.1098909201 \quad 1.5802537601$

C $0.0235025107-0.0116169669-1.3065989074$

F $-0.5249514106-1.1361515137-1.5799701665$

F $1.27640109170 .0678680479-1.5596507839$

F $-0.6666962616 \quad 1.0258792945-1.6021242158$

28ts chf3h, hydride ts, DFT

H $0.0044319577 \quad 0.00259258520 .000637843$

C $-0.02392229580 .0127383174 \quad 1.3304138522$

F $-0.7296287079 \quad 1.0556758477 \quad 1.5871913246$

F $1.22121419750 .100811607 \quad 1.6360381368$

F $-0.5812312508-1.111829477 \quad 1.6070627473$

C $0.0251158255-0.0120703501-1.3311243075$

F $-0.5242846763-1.1406196758-1.6063011822$

F $1.28081815560 .0681881195 \quad-1.5913444491$

F $-0.6681758443 \quad 1.027040202-1.632243812$

29d chloroform, $\mathrm{HF}$

H $0.00000051650 .-1.5224496848$

C $0.00000019660 .-0.4512291612$

Cl $1.6813971992 \quad 0.0 .0829384243$

Cl $-0.8406986495 \quad-1.45613270390 .0829373952$

$\mathrm{Cl} \quad-0.8406986494 \quad 1.45613270390 .0829373952$

29d chloroform, MP2

H $0.00000060320 .-1.5344382087$

$\begin{array}{llll}\text { C } & 0.0000001758 & 0 . & -0.449338424\end{array}$

Cl 1.68327149440 .0 .0829510171

Cl $-0.841635796 \quad-1.45775592440 .082950043$

Cl $-0.841635796 \quad 1.4577559244 \quad 0.082950043$

29d chloroform, DFT

H $-0.00004411330 .-1.5356764256$

$\begin{array}{llll}\text { C } & 0.000077885 & 0 . & -0.4534033248\end{array}$

Cl $1.7038337317 \quad 0.0 .0834672179$

$\begin{array}{lllll}\mathrm{Cl} & -0.8519293128 & -1.4756075192 & 0.0834456962\end{array}$

$\mathrm{Cl} \quad-0.8519293128 \quad 1.4756075192 \quad 0.0834456962$

29a $\mathrm{CCl} 3+, \mathrm{HF}$

Cl -0.0000001425 0. -1.6435208932

C 0. 0. 0 .

Cl $1.4233309165 \quad 0.0 .8217603232$

$\mathrm{Cl}-1.4233307739$ 0. 0.8217605701 

29a $\mathrm{CCl} 3+, \mathrm{MP} 2$
$\mathrm{Cl}-0.0000001428$ 0. -1.64719796
C 0. 0. 0 .
Cl 1.4265153499 0. 0.8235988563
$\mathrm{Cl}-1.426515207 \quad 0.0 .8235991037$
29a $\mathrm{CCl} 3+, \mathrm{DFT}$
Cl -0.000000144 0. -1.6601962622
C 0. 0. 0 .
Cl $1.4377722103 \quad 0.0 .8300980064$
$\mathrm{Cl}-1.4377720664 \quad 0.0 .8300982558$

29ts $\mathrm{CHCl} 3$, hydride transfer ts, $\mathrm{HF}$

H $0.0000461724 \quad 0.000014037 \quad 0.0000000671$

C $-0.0001195804-0.0010315062 \quad 1.3102380011$

$\begin{array}{lllll}\mathrm{Cl} & 1.4109724592 & 0.8973658717 & 1.6503699577\end{array}$

Cl 0.0725368455 - $1.6727138427 \quad 1.6481573574$

Cl $-1.483995756 \quad 0.7714422892 \quad 1.6499214593$

C $0.00013634570 .0010365399-1.3102379605$

Cl $-1.4110110634-0.8973045892-1.650289807$

Cl $1.4839617844-0.7715087565-1.6499805653$

Cl $-0.0724729028 \quad 1.6727164251 \quad-1.6481784203$

29ts $\mathrm{CHCl} 3$, hydride transfer cx, MP2

H 0.00003378910 .00035250260 .000060013

C $-0.0104897464 \quad-0.0300908186 \quad 1.2916748999$

$\begin{array}{lllll}\mathrm{Cl} & 0.1909299104 & 1.6268829502 & 1.6532445974\end{array}$

Cl 1.3266151198 -1.0467176394 1.5998328539

$\mathrm{Cl}-1.557094855 \quad-0.6929042508 \quad 1.584640842$

C $0.01050798790 .0300997587-1.2916384673$

Cl $-1.57068096550 .5899625108-1.6126843197$

Cl $0.3269418001-1.6098445359-1.6482164667$

Cl $1.2832805645 \quad 1.1325970743 \quad-1.5768338957$

29ts $\mathrm{CHCl} 3$, hydride transfer ts, DFT

H 0.00025910160 .00078566080 .0001268168

C $0.0007002853 \quad 0.0001997641 \quad 1.3437825461$

Cl $0.7803243057 \quad 1.4995344078 \quad 1.693476562$

Cl $0.9095275284-1.42462153831 .6923177642$

Cl $-1.6875411983-0.07457644951 .6934774163$

C $-0.00052552560 .0000244291 \quad-1.3439534296$

Cl $-1.6892063826-0.0722201924-1.6918856011$

Cl $0.9057918244-1.426276972-1.6929020571$

Cl $0.7810270013 \quad 1.4980354019$-1.6944312324

30d tetraflouroethylene, HF 
F $1.0784065666 \quad 0 .-1.3659389386$

C 0. 0. -0.6498083032

C 0. 0.0 .6498083032

F -1.0784065666 0. -1.3659389386

F $1.0784065666 \quad 0.1 .3659389386$

F -1.0784065666 0. 1.3659389386

30a tetraflouroethylene, MP2

F 1.1023396504 0. -1.38594236

C 0. 0. -0.6643835774

C 0.0 .0 .6643835774

F $-1.1023396504 \quad 0 .-1.38594236$

F 1.10233965040 .1 .38594236

F $-1.1023396504 \quad 0.1 .38594236$

30a tetraflouroethylene, DFT

F $1.10246959720 .-1.3890655016$

C 0. 0. -0.6612386122

C 0. 0.0 .6612386122

F $-1.10246959720 .-1.3890655016$

F 1.1024695972 0. 1.3890655016

F - 1.1024695972 0. 1.3890655016

30ts tetrafluoroethyl, hydride transfer ts, HF H $0.00033156190 .02717897 \quad-0.0610170664$

C $-1.2811119262-0.2963662453-0.3721566901$

F -1.89650497580 .25265107340 .6452423696$

F $-0.7033291013-2.1920617037-1.530565532$

F $-1.462691820 .4362880852-1.4440388521$

F $-1.158220733-2.38259836260 .5820354106$

C $-1.4955788313-1.7037909247 \quad-0.5421079168$

$\begin{array}{llll}\text { C } & 1.2833132841 & 0.4694122396 & -0.0169720738\end{array}$

F $1.891229579 \quad-0.6563389126 \quad 0.2630170427$

C $1.4934368126 \quad 1.53760228430 .9159614799$

$\begin{array}{llll}\text { F } & 1.4783172373 & 0.7734479629 & -1.2772371903\end{array}$

F 0.70847582282 .6018172460 .6087395333

F 1.14264759131 .15920315662 .1697703594

30ts tetrafluoroethyl', hydride transfer ts, MP2 H $0.000416496-0.0000973948-0.000021798$

C $-1.268458506-0.4226839957-0.1928369641$

F $-1.7530789822 \quad-0.2965689033 \quad 1.0770982963$

F - $-1.0361240921-1.8507197042-2.0364076655$

F $-1.74602523770 .6076347358-0.9503803458$

F - $-1.0430657454-2.7490640994-0.0221420302$

C $-1.5225277961-1.7132445731 \quad-0.7692982339$ 
C 1.26874690240 .42223227850 .1937780174

F $1.74520413-0.6069276470 .9535857133$

C $1.5223252372 \quad 1.71375760920 .7684331454$

F $1.7550308643 \quad 0.2939257345 \quad-1.0753046204$

F $1.0438836313 \quad 2.748341442 \quad 0.0188727816$

F $1.0340719295 \quad 1.85334838392 .0346296495$

30ts tetrafluoroethyl, hydride transfer ts, DFT H $0.00035713910 .0328883606-0.073896361$

C $-1.288446364-0.370981634-0.3122537906$

F $-1.8698468383 \quad 0.02041993730 .8558989054$

F $-0.9211568052-2.1426098881-1.8082297524$

F $-1.6463589410 .5037442563-1.2934008036$

F $-1.1584458064 \quad-2.62374580590 .3382998273$

$\begin{array}{llll}\text { C } & -1.5421788043 & -1.7476444292 & -0.6475930804\end{array}$

$\begin{array}{llll}\text { C } & 1.2896056826 & 0.4755518242 & 0.0771282606\end{array}$

F $1.8627361465 \quad-0.6561498046 \quad 0.5736826857$

C $1.5410487153 \quad 1.64567550980 .8768939012$

F $1.65886200710 .6169043827 \quad-1.2265518583$

F $0.9280716488 \quad 2.77429898980 .3878001958$

F $1.1460794199 \quad 1.5017494897 \quad 2.1845946463$

31 tetrafluoroethyl + , HF

H $0.79383843140 .3723280221 \quad-1.4711555157$

$\begin{array}{llll}\text { C } & 0.4469071865 & 0.5929000214 & -0.4663106453\end{array}$

F 1.43592633590 .80626901910 .3511160825

$\begin{array}{llll}\text { C } & -0.3027310259 & -0.6642902212 & 0.0678643012\end{array}$

F $-0.4146697069 \quad 1.5672848608 \quad-0.4548673484$

F $0.2852660187-1.5488582105 \quad 0.6531916239$

F $-1.4908443582-0.818471983-0.120347738$

31 tetrafluoroethyl +, MP2

$\begin{array}{lllll}\text { H } & 0.8006504408 & 0.3730763191 & -1.4860856758\end{array}$

$\begin{array}{lllll}\text { C } & 0.4475900546 & 0.5910431008 & -0.4696311576\end{array}$

$\begin{array}{lllll}\text { F } & 1.459218545 & 0.8074662829 & 0.3610261187\end{array}$

C $-0.3019346913-0.6657917975 \quad 0.0646181546$

F $-0.4312893042 \quad 1.5848947737 \quad-0.4623425803$

$\begin{array}{lllll}\text { F } & 0.2990968435 & -1.5646025411 & 0.66284654\end{array}$

F $-1.51309082-0.8193789754-0.1264007791$

31 tetrafluoroethyl +, DFT

H $0.7974007207 \quad 0.3678372502 \quad-1.4835740367$

$\begin{array}{lllll}\text { C } & 0.4467035974 & 0.5954870031 & -0.4634004636\end{array}$

$\begin{array}{lllll}\text { F } & 1.4670149991 & 0.8167651612 & 0.3594949071\end{array}$

$\begin{array}{llll}\text { C } & -0.304307613 & -0.6674522665 & 0.0684988693\end{array}$

F $-0.4283584922 \quad 1.596194501 \quad-0.4659909386$ 
F $0.2956231748-1.57579434220 .6621993406$

F $-1.517810418-0.8300592832-0.1275940199$

31ts intra tetrafluoroethyl + , hydride shift ts, HF H 0. 0. 1.2131516084

F $-1.344165543-1.0694799648-0.0401868706$

F $1.344165543-1.0694799648-0.0401868706$

C -0.69448580690 .0 .0194646444$

$\begin{array}{llll}C & 0.6944858069 & 0.0 .0194646444\end{array}$

F $-1.344165543 \quad 1.0694799648-0.0401868706$

F $1.344165543 \quad 1.0694799648 \quad-0.0401868706$

31ts intra tetrafluoroethyl+, hydride shift ts, MP2 H 0. 0. 1.2160673722

F - $-1.3624614285-1.0914729847-0.0396961294$

F $1.3624614285-1.0914729847-0.0396961294$

C $-0.7052630075 \quad 0.0 .0177494404$

$\begin{array}{llll}C & 0.7052630075 & 0.0 .0177494404\end{array}$

F $-1.3624614285 \quad 1.0914729847 \quad-0.0396961294$

F $1.3624614285 \quad 1.0914729847 \quad-0.0396961294$

31ts intra tetrafluoroethyl + , hydride shift ts, DFT H 0. 0. 1.2430078374

F - $-1.36734666666-1.0917284268-0.0419910179$

F $1.36734666666-1.0917284268-0.0419910179$

C -0.705683657110 .0 .0223890673$

$\begin{array}{llll}C & 0.7056836571 & 0.0 .0223890673\end{array}$

F $-1.3673466666 \quad 1.0917284268 \quad-0.0419910179$

F $1.36734666661 .0917284268-0.0419910179$

32 1,1,2,2-tetramethylethyl+, HF

H $-1.18781319611 .1137007321-1.8534856495$

C $-0.12862859640 .9085298291 \quad-1.7401780928$

$\begin{array}{llll}\text { H } & 0.1224862976 & 0.0340145372 & -2.3288598424\end{array}$

$\begin{array}{llll}\text { C } & 0.2611321133 & 0.7537492329 & -0.2681720295\end{array}$

H $0.41373809341 .7496075559-2.1540496042$

C $-0.4237181218-0.3171474571 \quad 0.480918281$

H $0.0990097559 \quad 1.686274074 \quad 0.2621950551$

C $1.77923851580 .3963915979 \quad-0.1042134891$

H $2.0267500025 \quad-0.5273751263-0.6139244364$

H 2.08261568190 .32449415380 .9333764223

H $2.34025459331 .1997237598 \quad-0.5639016716$

C $-0.6589156016-0.1577893443 \quad 1.926437943$

C $-0.8978080631-1.5431823075-0.1876239241$

H $-1.1164260022 \quad-2.3438518633 \quad 0.5032440366$

H $-0.2127772346-1.8666719183 \quad-0.9616273331$ 


$$
\begin{array}{llll}
\text { H } & -1.821063376 & -1.2547161222 & -0.6981803586 \\
\text { H } & -0.1175338884 & 0.6700437915 & 2.3600991477 \\
\text { H } & -0.4855259031 & -1.0807121077 & 2.4669869252 \\
\text { H } & -1.7315163012 & 0.0521592273 & 2.0051151776 \\
\text { 32 } & & 1,1,2,2 \text {-tetramethylethyl, } & \text { MP } 2 \\
\text { H } & -0.9858825377 & 1.6048291114 & -1.6781647946 \\
\text { C } & -0.5733784723 & 1.7828061641 & -0.6814776235 \\
\text { H } & 0.4528055097 & 2.1438901939 & -0.782069749 \\
\text { C } & -0.6574875204 & 0.5245907236 & 0.1736832889 \\
\text { H } & -1.1607071508 & 2.5727075218 & -0.2079562548 \\
\text { C } & 0.0916234139 & -0.6428059558 & -0.2327203163 \\
\text { H } & -1.6885578975 & 0.251727981 & 0.4186240767 \\
\text { C } & 0.0750895254 & 0.7401608063 & 1.5797541698 \\
\text { H } & 1.1294522727 & 0.9891594153 & 1.444152216 \\
\text { H } & -0.0322265327 & -0.1126422026 & 2.2518196235 \\
\text { H } & -0.4416415257 & 1.6014111363 & 2.0118006818 \\
\text { C } & -0.3373160782 & -1.9817289763 & 0.1998623495 \\
\text { C } & 1.2931596135 & -0.5225614062 & -1.0722854072 \\
\text { H } & 1.9354961572 & -1.4020136725 & -1.0357007063 \\
\text { H } & 1.8503724237 & 0.39708068 & -0.8796798084 \\
\text { H } & 0.8972740166 & -0.4171408609 & -2.0997836895 \\
\text { H } & -1.196153231 & -1.972202962 & 0.8700427622 \\
\text { H } & 0.501354767 & -2.5501803183 & 0.6167866851 \\
\text { H } & -0.6117291625 & -2.509394158 & -0.7307698102
\end{array}
$$

$$
\begin{array}{llll}
\text { 32 } & \multicolumn{4}{c}{1,1,2,2 \text {-tetramethylethyl+, DFT }} \\
\text { H } & -0.953786198 & 1.5771973121 & -1.6815141295 \\
\text { C } & -0.5573750098 & 1.7732469612 & -0.6827264456 \\
\text { H } & 0.4574706081 & 2.1638639639 & -0.7800609095 \\
\text { C } & -0.6240473752 & 0.5238122097 & 0.1984514587 \\
\text { H } & -1.1675590444 & 2.5575693492 & -0.232397791 \\
\text { C } & 0.1085689945 & -0.6605139092 & -0.2426359402 \\
\text { H } & -1.6609821101 & 0.2408472779 & 0.4070549929 \\
\text { C } & 0.0308395256 & 0.7959908173 & 1.6264158141 \\
\text { H } & 1.0801370642 & 1.0768633603 & 1.5323113519 \\
\text { H } & -0.065721435 & -0.050228656 & 2.3059242546 \\
\text { H } & -0.5240164468 & 1.6399799931 & 2.0396259697 \\
\text { C } & -0.3279814587 & -1.9933662514 & 0.1904714687 \\
\text { C } & 1.2828047998 & -0.547618976 & -1.115360963 \\
\text { H } & 1.8936185706 & -1.4477369585 & -1.1518840005 \\
\text { H } & 1.883856943 & 0.3367681496 & -0.8861741689 \\
\text { H } & 0.8877511552 & -0.3531441264 & -2.1288670078 \\
\text { H } & -1.1417484655 & -1.9831608572 & 0.9131923298 \\
\text { H } & 0.514433258 & -2.6012516429 & 0.5373572327 \\
\text { H } & -0.6803107566 & -2.5068722742 & -0.7222604803
\end{array}
$$


32ts intra 1,1,2,2-tetramethylethyl+, H-shift ts, HF

H $0.2848282878 \quad 2.71712505 \quad 0.3988253489$

H $2.3569885829-0.2643862276-0.0039007207$

H $2.2470772181 \quad 1.34471713370 .7141524769$

C $1.85368136110 .6866060685 \quad-0.0504953973$

C $-0.3498014073-0.6072455406 \quad 0.0514090368$

C $0.3498014073 \quad 0.6072455406 \quad 0.0514090368$

H 0.0 .1 .171422725

H $2.07045851451 .1423799879-1.0128780701$

H $-1.3007895786 \quad 1.96834533640 .4903471724$

H $-0.51487849012 .1791738564-1.0671939951$

H $-2.2470772181-1.34471713370 .7141524769$

C $-0.3498014073 \quad 1.9477254144 \quad-0.0184242352$

C $0.3498014073-1.9477254144-0.0184242352$

C $-1.8536813611-0.6866060685-0.0504953973$

$\mathrm{H}-2.0704585145-1.1423799879-1.0128780701$

H $0.5148784901-2.1791738564-1.0671939951$

H $-0.2848282878 \quad-2.71712505 \quad 0.3988253489$

H $1.3007895786 \quad-1.96834533640 .4903471724$

H $-2.35698858290 .2643862276-0.0039007207$

32ts intra 1,1,2,2-tetramethylethyl + , H- shift (not a ts)

$\mathrm{H}-1.0713673503 \quad-2.523408594 \quad-0.402239596$

H $2.1944946676-0.891711495 \quad 0.0279343121$

H $1.3288157864-2.2633472645 \quad-0.7232270959$

C $1.2899442983-1.49439115940 .0520246418$

C $-0.01144692960 .7081390907-0.0482077421$

C $0.0114469296-0.7081390907-0.0482077421$

H $0.0 .-1.1583721973$

H $1.2440485092-2.01305440331 .0172351015$

H - $2.0954623342-1.0766529427-0.5063311861$

H $-1.5195940878-1.6455024861 .0692901819$

H $-1.32881578642 .2633472645 \quad-0.7232270959$

C $-1.2522579832-1.53084178390 .0122704973$

C $1.2522579832 \quad 1.53084178390 .0122704973$

C $-1.2899442983 \quad 1.4943911594 \quad 0.0520246418$

H - $1.2440485092 \quad 2.0130544033 \quad 1.0172351015$

H $1.5195940878 \quad 1.6455024861 .0692901819$

H $1.07136735032 .523408594-0.402239596$

H $2.0954623342 \quad 1.0766529427 \quad-0.5063311861$

H $-2.1944946676 \quad 0.891711495 \quad 0.0279343121$

32ts intra 1,1,2,2-tetramethylethyl+, H- shift ts, DFT

$\mathrm{H}-1.1004138355-2.5022959174-0.4594908169$

H $2.1970455626-0.9178358245-0.0682585487$ 
H $1.2881165769-2.3230823564-0.6727217901$

C $1.2875514652-1.5004150840 .0441978251$

C $-0.0104908637 \quad 0.7091529625-0.0511821409$

C $0.0104908637-0.7091529625-0.0511821409$

H 0. 0. -1.1751913895

H $1.2992990642-1.95270838961 .0428060052$

H $-2.1243215425-1.0566873402-0.4135728998$

H - $1.4615369004-1.7217360753 \quad 1.0793284476$

H $-1.28811657692 .3230823564-0.6727217901$

C $-1.2497963585-1.535337208 \quad 0.0202351987$

C $1.2497963585 \quad 1.535337208 \quad 0.0202351987$

C $-1.2875514652 \quad 1.5004150840 .0441978251$

H - $1.29929906421 .9527083896 \quad 1.0428060052$

H 1.46153690041 .72173607531 .0793284476

H $1.10041383552 .5022959174 \quad-0.4594908169$

H $2.1243215425 \quad 1.0566873402-0.4135728998$

H $-2.19704556260 .9178358245 \quad-0.0682585487$ 